\title{
Analysis of the acoustic cut-off frequency and high-frequency peaks in six Kepler stars with stochastically excited pulsations ${ }^{\star}$
}

\author{
A. Jiménez ${ }^{1,2}$, R. A. García ${ }^{3}$, F. Pérez Hernández ${ }^{1,2}$, and S. Mathur ${ }^{4}$ \\ 1 Instituto de Astrofísica de Canarias, 38205 La Laguna, Tenerife, Spain \\ e-mail: ajm@iac.es \\ 2 Universidad de La Laguna, Dpto. de Astrofísica, 38206 Tenerife, Spain \\ 3 Laboratoire AIM, CEA/DSM - CNRS - Univ. Paris Diderot - IRFU/SAp, Centre de Saclay, 91191 Gif-sur-Yvette Cedex, France \\ ${ }^{4}$ Space Science Institute, 4750 Walnut Street, Suite 205, Boulder, CO 80301, USA
}

Received 5 May 2015 / Accepted 12 August 2015

\section{ABSTRACT}

\begin{abstract}
Gravito-acoustic modes in the Sun and other stars propagate in resonant cavities with a frequency below a given limit known as the cut-off frequency. At higher frequencies, waves are no longer trapped in the stellar interior and become traveller waves. In this article, we study six pulsating solar-like stars at different evolutionary stages observed by the NASA Kepler mission. These high signal-tonoise targets show a peak structure that extends at very high frequencies and are good candidates for studying the transition region between the modes and interference peaks or pseudo-modes. Following the same methodology successfully applied on Sun-as-a-star measurements, we uncover the existence of pseudo-modes in these stars with one or two dominant interference patterns depending on the evolutionary stage of the star. We also infer their cut-off frequency as the midpoint between the last eigenmode and the first peak of the interference patterns. Using ray theory we show that, while the period of one of the interference patterns is very close to half the large separation, the period of the other interference pattern depends on the time phase of mixed waves, thus carrying additional information on the stellar structure and evolution.
\end{abstract}

Key words. asteroseismology - stars: evolution - stars: solar-type - stars: oscillations

\section{Introduction}

Solar-like oscillation spectra are usually dominated by $p$-mode eigenfrequencies corresponding to waves trapped in the stellar interior with frequencies below a given cut-off frequency, $v_{\text {cut }}$. However, the oscillation power spectrum of the resolved Sun shows a regular peak structure that extends well above $v_{\text {cut }}$ (e.g. Jefferies et al. 1988; Libbrecht 1988; Duvall et al. 1991). This signal is interpreted as travelling waves whose interferences produce a well-defined pattern corresponding to the so-called pseudo-mode spectrum (Kumar et al. 1990). The amount and quality of the space data provided by the Solar and Heliospheric Observatory (SoHO) satellite (Domingo et al. 1995) allowed us to measure these high-frequency peaks (HIPs), using Sun-as-astar observations (García et al. 1998) from Global Oscillations at Low Frequency (GOLF, Gabriel et al. 1995) and from Variability of solar IRradiance and Gravity Oscillations (VIRGO, Fröhlich et al. 1995) instruments. The change in the frequency pattern between the acoustic and the pseudo-modes enabled the proper determination of the solar cut-off frequency (Jiménez 2006).

Theoretically, the cut-off frequency approximately scales as $g \sqrt{\mu / T_{\text {eff }}}$, where $T_{\text {eff }}$ is the effective temperature, $g$ the gravity, and $\mu$ the mean molecular weight, with all values measured at the surface. Hence, the observed $v_{\text {cut }}$ can be used to constrain the fundamental stellar parameters, however, solar observations showed that $v_{\text {cut }}$ changes, for example, with the solar magnetic activity cycle (Jiménez et al. 2011). Therefore, the accuracy of

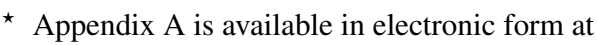
http: //www . aanda.org
}

the scaling relation given above needs to be discussed in a theoretical context and taking observational constraints into account.

For stars other than the Sun, the detection of HIPs is challenging because of the shorter length of available data sets and lower signal-to-noise ratio $(\mathrm{S} / \mathrm{N})$. Nevertheless, in this study we report on the analysis of the high-frequency part of the spectrum of six pulsating stars observed by Kepler (Borucki et al. 2010) for which we were able to characterize the HIP pattern and the cut-off frequency. The time series analysis and preparation of the spectra are detailed in Sect. 2. In Sect. 3 we describe how to estimate $v_{\text {cut }}$ and compare it with our theoretical expectations. In Sect. 4 we perform a detailed analysis of the HIPs and interpret the results as a function of the evolutionary stage of the stars. Finally, we provide our conclusions in Sect. 5.

\section{Data analysis}

In this study, we used ultra high-precision photometry obtained by NASA's Kepler mission to study the high-frequency region of six stars with solar-like pulsations. The names of the stellar identifiers from the Kepler Input Catalogue (KIC, Brown et al. 2011) are given in the first column of Table 1 . Short-cadence time series (sampling rate of 58.85s, Gilliland et al. 2010) up to quarter 17 have been corrected for instrumental perturbations and properly stitched together using the Kepler Asteroseismic Data Analysis and Calibration Software (KADACS, García et al. 2011).

For each star, the long time series (approximately four years) are divided into consecutive subseries and the average of all 

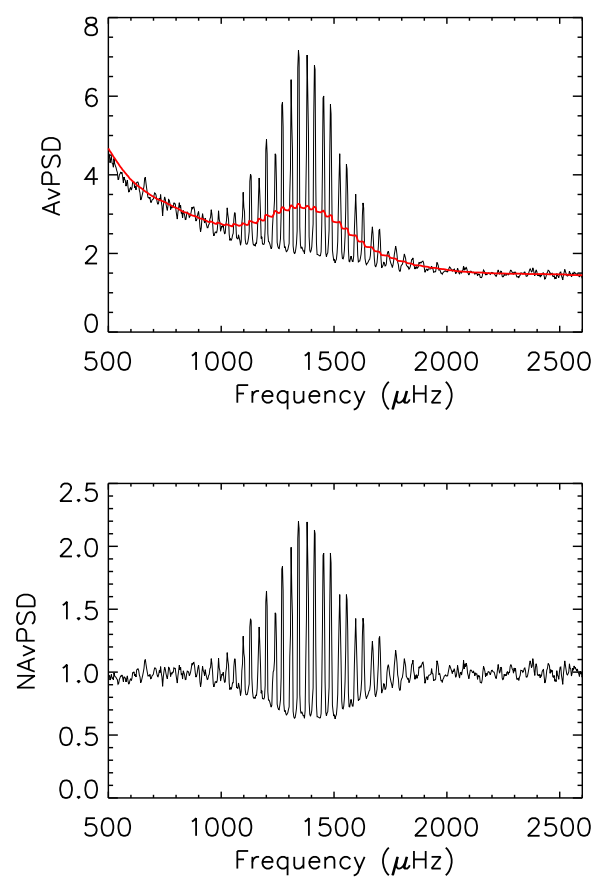

Fig. 1. Top panel: smoothed averaged power spectral density of fourday subseries of KIC 11244118. The red line is the severe smoothing used to normalize the spectrum (NAvPSD) shown in the lower panel (see text for details).

the power spectral density (AvPSD) is computed to reduce the high-frequency noise in the spectrum. As in the solar case (e.g. García et al. 1998; Jiménez et al. 2005, 2006), subseries of approximately four-days $(4 \times 1440$ points, 3.92 days $)$ were used because they are a good compromise between frequency resolution (which improves with longer subseries) and the increase of the $\mathrm{S} / \mathrm{N}$ (which improves with the number of averaged spectra). For the same reasons as in the solar case, we use a boxcar function to smooth the AvPSD over 3 or 5 points, depending on the evolutionary state of the star and the $\mathrm{S} / \mathrm{N}$ of the spectrum. From now on when we refer to the AvPSD, we mean the smoothed AvPSD. An example of this spectrum is given in the top panel of Fig. 1 for KIC 11244118. Because of the short length of the subseries, it is not necessary to interpolate the gaps in the data (see for more details García et al. 2014), so we prefer the simplest possible analysis. However, we have verified that the results remain the same when using series that were interpolated with an inpainting algorithm (Pires et al. 2015).

To avoid subseries of low quality and to increase the $\mathrm{S} / \mathrm{N}$ in the AvPSD, we first remove all subseries with a duty cycle below $50 \%$. Then, for each subseries of each star we compute the median of the flat noise at high frequencies above 2 to $5 \mathrm{mHz}$, depending on the frequency of maximum power of the star. From the statistical analysis of these medians, we reject those subseries in which the high-frequency noise is too high. We have verified that the selection of different high-frequency ranges does not affect the number of series retained. A detailed study of the rejected series show that there is an increase in the noise level when Kepler has lost the fine pointing and the spacecraft is in "coarse" pointing mode. This is particularly important in Q12 and Q16. A new revision of the KADACS software (Mathur, Bloemen, García, in prep.) systematically removes those data points from the final corrected time series. In Table 1 we summarize the number of four-day subseries computed for each star, the number of subseries finally retained for the calculation of the
Table 1. Stars and time series used in this study.

\begin{tabular}{lccc}
\hline \hline KIC & \#Series & \#Averaged series & Qi-Qf \\
\hline 3424541 & 292 & 270 & Q5-Q17 \\
7799349 & 292 & 268 & Q5-Q17 \\
7940546 & 244 & 184 & Q7-Q17 \\
9812850 & 292 & 276 & Q5-Q17 \\
11244118 & 292 & 265 & Q5-Q17 \\
11717120 & 292 & 276 & Q5-Q17 \\
\hline
\end{tabular}

Notes. Columns: KIC, the name of each star; number of initial four-day subseries; number of finally averaged subseries after removing those with a low $\mathrm{S} / \mathrm{N}$; and the initial and final Kepler short-cadence quarters used in the analysis.

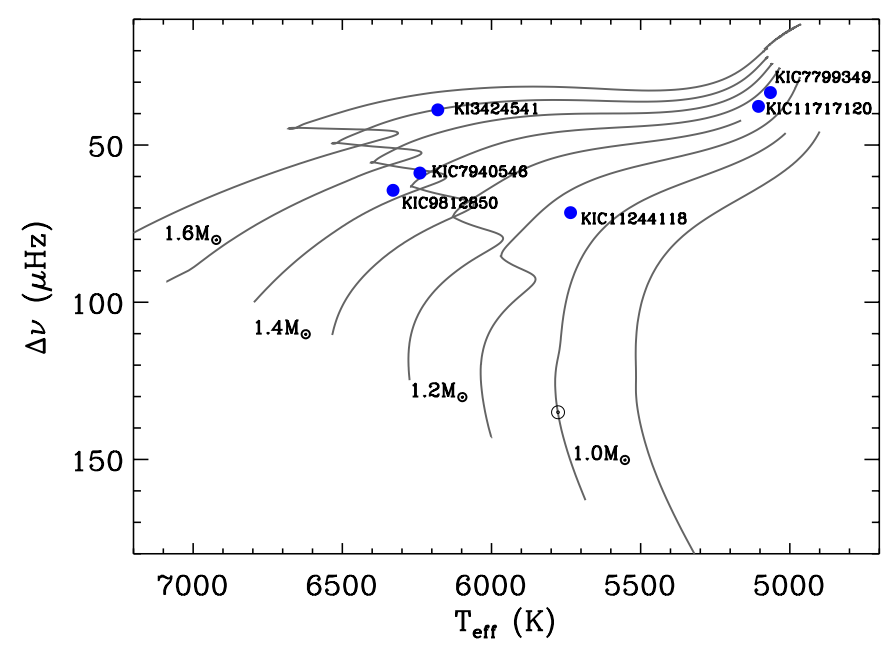

Fig. 2. Seismic HR diagram showing the position of the six stars analysed. The position of the Sun is indicated by the $\odot$ symbol. The evolutionary tracks are computed using ASTEC (Christensen-Dalsgaard 2008).

AvPSD, and the total number of short-cadence Kepler quarters available.

Figure 2 shows the place of the observed stars in a seismic HR diagram. The black lines are the evolution sequences computed using the Aarhus Stellar Evolution Code (ASTEC; Christensen-Dalsgaard 2008) in a range of masses from $M=$ $0.9 M_{\odot}$ to $M=1.6 M_{\odot}$ in steps of $M=0.1 M_{\odot}$ with a solar composition $\left(Z_{\odot}=0.0246\right)$. The target stars cover evolutionary stages from late main-sequence stars to early red giants.

\section{The acoustic cut-off frequency}

\subsection{Observations}

The observed stellar power spectrum has different patterns in the eigenmode and pseudo-mode regions. In particular, the mean frequency separation, $\langle\Delta v\rangle$, between consecutive peaks is different below and above the cut-off frequency. In the first case, below the cut-off frequency corresponds to half the mean large frequency spacing, while above $v_{\text {cut }}$ it is the period of the interference pattern. In addition, a phase shift between both patterns appears in the transition region. The frequency in which the transition between the two regimes is observed corresponds to the cut-off frequency. Hence, we fit all the peaks in the spectrum 
(actually doublets of odd, $\ell=1,3$, and even, $\ell=0,2$, pairs of modes due to the small frequency resolution of $2.89 \mu \mathrm{Hz}$ ) from a few orders before $v_{\max }$ up to the highest visible peak in the spectrum. To account for the underlying background contribution properly, which is due to convective movements at different scales, faculae, and magnetic/rotation signal, we prefer to divide the AvPSD by the same spectrum after heavy smoothing (top panel of Fig. 1) instead of using a theoretical model (e.g. Mathur et al. 2011). Although it has been demonstrated that a two-component model usually properly fits the background of stars (for more details, see Kallinger et al. 2014), the accuracy in the transition region between the eigenmode bump and the high-frequency region dominated by the HIPs is not properly described. For the purposes of this study, we are primarily interested in the transition region. Therefore, we use a simpler description of the background based on the observed spectrum itself. The length of this smoothing varies according to the evolutionary stage of the star. An example of the normalized resultant AvPSD (NAvPSD) is given in the bottom panel of Fig. 1.

A global fit to all the visible peaks in the spectrum is not possible for the following reasons: 1) it requires a huge amount of computation time owing to the high number of free parameters in the model; 2) the background is not completely flat around $v_{\max }$; and 3) the large amplitude dispersion of the peaks between $v_{\max }$ and the HIP pattern biases the results of the smallest peaks by overestimating their amplitudes. We therefore divide the spectrum into a few orders before $v_{\max }$ and the photonnoise dominated region into two regions. We arbitrarily choose a frequency where the amplitudes start to be very small and separately treat the power spectrum before and after this frequency. We denote, with $p$-mode region, the low-frequency zone before the frequency mentioned above and the pseudo-modes region is the one after that frequency. Several tests have been performed in which the frequency separating these two regions were varied and in all cases the results remain the same.

In the $p$-mode region, we fit groups of several peaks at a time for which the underlying background can be considered flat. This fit depends on the evolutionary stage of the star and $\mathrm{S} / \mathrm{N}$. We checked that fitting a different number of peaks in each group does not change the final result. Appendix A shows the analysis of all the stars, and in the caption of each figure we explicitly mention the number of peaks fitted together. The pseudo-modes region can be fitted at once because 1) the background is flat (see the bottom panel of Fig. 1);2) the range of the peak amplitudes is reduced; and 3) the number of free parameters is small. For both zones, $p$-mode and pseudo-mode, a Lorentzian profile is used to model the peaks using a maximum likelihood estimator. We are only interested in the frequency of the centroids of the peaks, and for that a Lorentzian profile is a good approximation. In Figs. 3 and 4 we show the results of the fits for KIC 3424541 in the $p$-mode and pseudo-mode regions. The figures for the other stars can be found in Appendix A.

After fitting the two parts of the spectra, we compute the frequency differences of consecutive peaks: $v_{n}-v_{n-1}$. As an example, the resultant frequency differences for KIC 3424541 are plotted in Fig. 5.

Two different regions are clearly visible in the frequency differences of KIC 3424541. A portion of these differences, those at lower frequencies, are centred around $20.75 \mu \mathrm{Hz}$, corresponding approximately to half of the large spacing, $\Delta v_{\text {modes }} / 2$ (see Table 2) and owing to the alternation between odd and even modes. At a certain frequency, between 1200 and $1230 \mu \mathrm{Hz}$, the frequency separations increase and remain roughly constant around $37.31 \mu \mathrm{Hz}$, although with a higher dispersion; see the
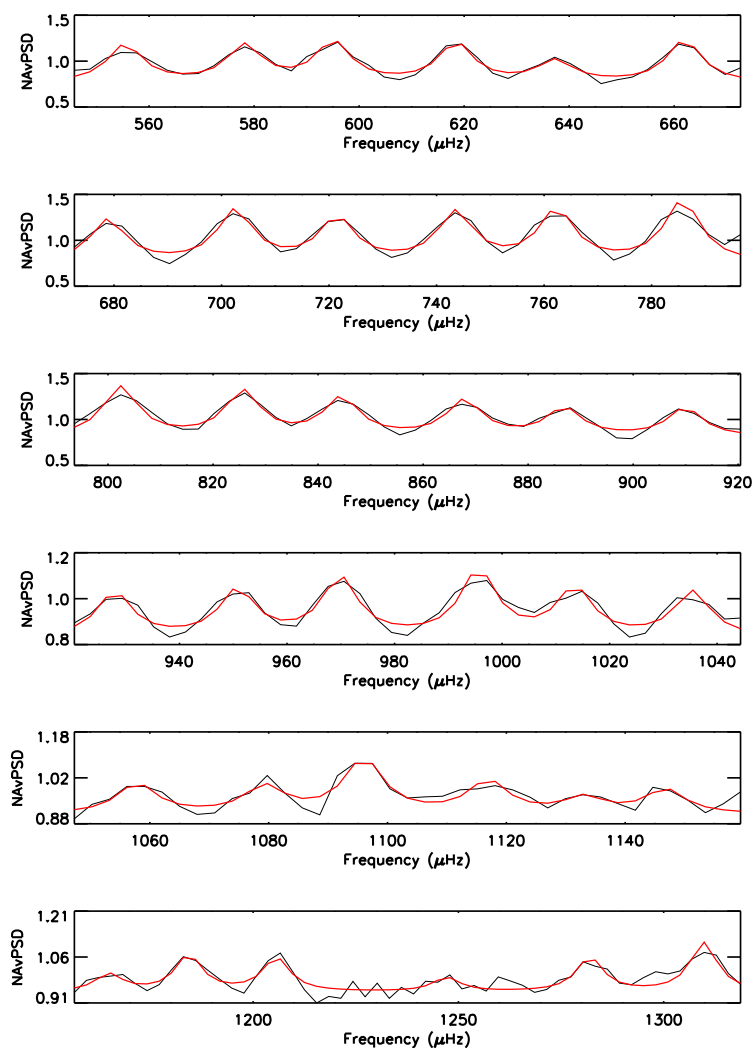

Fig. 3. Sections of the NAvPSD used to fit the eigenmode region in groups of six modes in KIC 3424541. The red line represents the final fit.

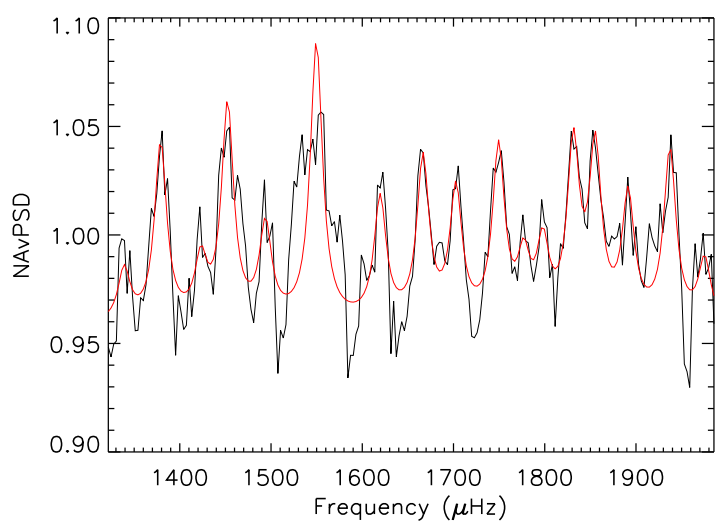

Fig. 4. Pseudo-mode region of the NAvPSD in KIC 3424541. The red line is the result of the fit.

next section for further details. The observed acoustic cut-off frequency, $v_{\text {cut }}$, lies in the transition region where the differences jump from the $p$-mode region to the pseudo-mode zone. We define this position as the mean frequency between the two closest points to this transition, represented by a red symbol in Fig. 5 for KIC 3424541. The results of this analysis for the six stars studied here are given in Table 2.

\subsection{Comparison between the observed and theoretical cut-off frequency}

As noted in Sect. 1, the cut-off frequency at the stellar surface scales approximately as $v_{\text {cut }} \propto g \sqrt{\mu / T_{\text {eff }}}$, suggesting that we 
Table 2. Stellar parameters I.

\begin{tabular}{lcccccccc}
\hline \hline KIC & $T_{\text {eff }}$ & $v_{\max }$ & $v_{\text {cut }}$ & $\Delta v_{\text {modes }}$ & $\Delta v_{1}$ & $\Delta v_{2}$ & $A_{1}$ & $A_{2}$ \\
\hline Sun (GOLF) & 5777 & $3097 \pm 116$ & $5106.41 \pm 61.53$ & $134.9 \pm 0.8$ & $70.29 \pm 0.65$ & $143.77 \pm 6.14$ & $0.018 \pm 0.020$ & $0.008 \pm 0.017$ \\
Sun (VIRGO) & 5777 & $3097 \pm 116$ & $5106.41 \pm 61.53$ & $134.9 \pm 0.8$ & $71.70 \pm 0.56$ & $151.16 \pm 14.33$ & $0.056 \pm 0.038$ & $0.010 \pm 0.024$ \\
3424541 & 6180 & $761.97 \pm 39.10$ & $1210.67 \pm 15.71$ & $38.8 \pm 1.2$ & $20.3 \pm 2.1$ & $39.8 \pm 1.5$ & $0.001 \pm 0.018$ & $0.011 \pm 0.016$ \\
7799349 & 5065 & $568.14 \pm 9.87$ & $895.47 \pm 15.38$ & $33.3 \pm 0.2$ & $19.3 \pm 2.5$ & $33.7 \pm 0.9$ & $0.002 \pm 0.017$ & $0.013 \pm 0.016$ \\
7940546 & 6240 & $1099.57 \pm 28.02$ & $1940.59 \pm 20.71$ & $58.9 \pm 0.4$ & $30.3 \pm 0.4$ & $48.9 \pm 1.0$ & $0.021 \pm 0.023$ & $0.020 \pm 0.021$ \\
9812850 & 6330 & $1224.38 \pm 52.21$ & $1898.08 \pm 24.29$ & $64.4 \pm 0.5$ & $31.8 \pm 1.6$ & $54.0 \pm 2.6$ & $0.009 \pm 0.024$ & $0.018 \pm 0.020$ \\
11244118 & 5735 & $1384.07 \pm 50.26$ & $1968.69 \pm 21.48$ & $71.5 \pm 0.5$ & $37.1 \pm 2.0$ & $54.8 \pm 3.4$ & $0.014 \pm 0.029$ & $0.018 \pm 0.025$ \\
11717120 & 5105 & $582.96 \pm 5.86$ & $958.38 \pm 11.56$ & $37.7 \pm 0.3$ & $16.6 \pm 1.3$ & $29.9 \pm 0.8$ & $-0.003 \pm 0.030$ & $0.030 \pm 0.026$ \\
\hline
\end{tabular}

Notes. Columns: KIC, the number of each star, effective temperature from Bruntt et al. (2012) with an error bar of $84 \mathrm{~K}$, $v_{\max }$ computed using the A2Z pipeline (Mathur et al. 2010), $v_{\text {cut }}, \Delta v_{\text {modes }}, \Delta v_{1}, \Delta v_{2}, A_{1}$, and $A_{2}$ computed as explained in the text.

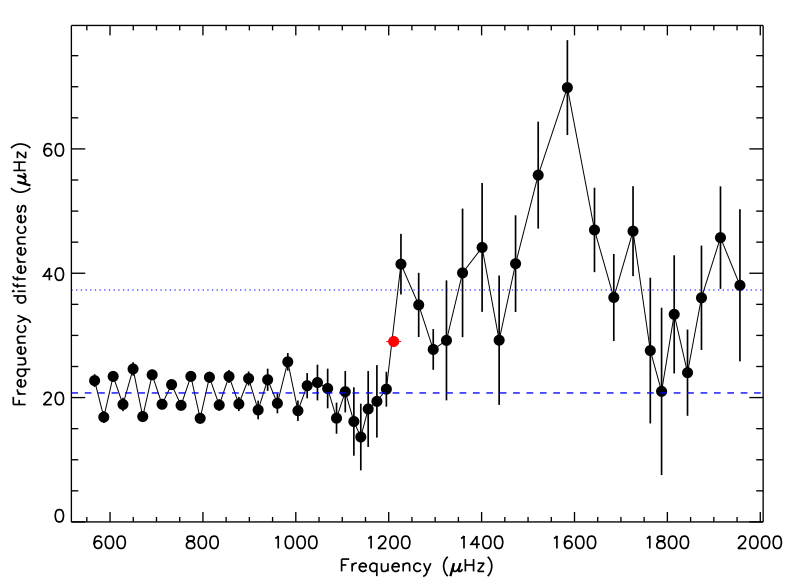

Fig. 5. Consecutive frequency differences of KIC 3424541. The blue dashed and dotted lines are the weighted mean value of the frequency differences in the $p$-mode $(20.75 \pm 0.20 \mu \mathrm{Hz})$ and pseudo-mode regions $(37.31 \pm 1.58 \mu \mathrm{Hz})$, respectively. The red symbol represents the estimated cut-off frequency, $v_{\text {cut }}=1210.67 \pm 15.70 \mu \mathrm{Hz}$.

may compare the observational cut-off frequency with that computed from the spectroscopic parameters. However, for our set of stars, the spectroscopic $\log g$ has large uncertainties (see Bruntt et al. 2012) and a direct test is not possible. Alternatively, it has been demonstrated empirically that the frequency of maximum mode amplitude $v_{\max }$ follows a similar scale relation (see Bedding \& Kjeldsen 2003). As is seen in Fig. 6, the observational data show a linear relation between $v_{\max }$ and $v_{\text {cut }}$, although some stars deviate $2 \sigma$ from it. In fact, from a theoretical point of view, one does not expect this linear relation to be accurate enough at the level of the observational errors. As shown below, values of $v_{\text {cut }}$ can deviate by as much as $10 \%$ from the scale relation $v_{\text {cut }} \propto g \sqrt{\mu / T_{\text {eff }}}$ for representative models of our stars. On the other hand, following Chaplin et al. (2008), where theoretical values of $v_{\max }$ based on stochastic mode excitation were computed, one can find departures from the scale relation as large as $25 \%$ for a model of a star with $M=1.3 M_{\odot}$ and $v_{\max } \sim 1500 \mu \mathrm{Hz}$.

We can gain a deeper insight by comparing the observational and theoretical results in the $v_{\text {cut }}-\Delta v$ plane. For the theoretical calculations, we use a set of models computed with the "Code d'Évolution Stellaire Adaptatif et Modulaire" (CESAM) code (Morel \& Lebreton 2008). These correspond to evolutionary tracks from the zero-age main sequence up to the red-giant

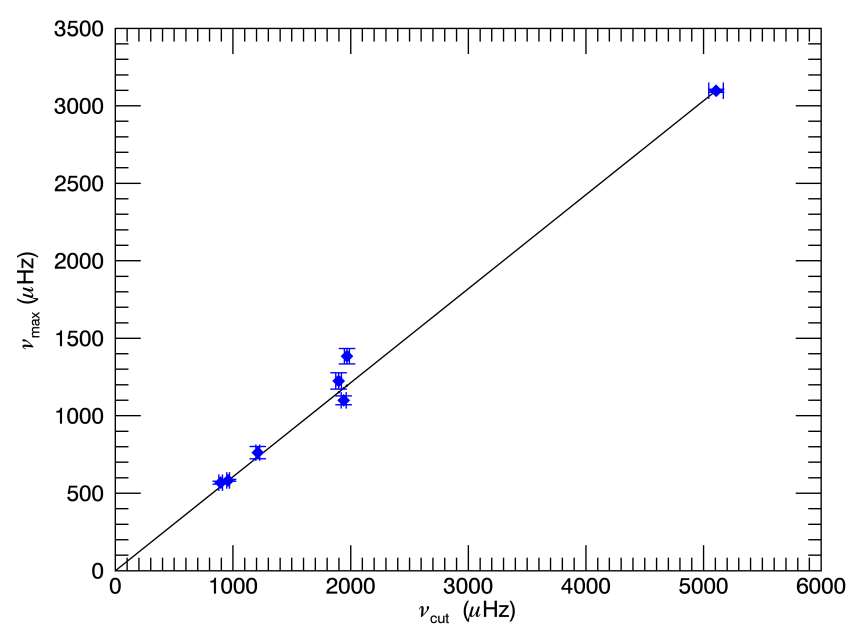

Fig. 6. Observed cut-off frequencies $v_{\text {cut }}$ versus maximum mode amplitude frequencies $v_{\max }$. Blue points with errors are values for the six stars considered here and the Sun (the rightmost point). A straight line through the Sun has been drawn for guidance.

phase, with masses between $M=0.9 M_{\odot}$ and $M=2.0 M_{\odot}$, and helium abundances between $Y=0.25$ and $Y=0.28$. Models with and without overshooting were considered. Other parameters were fixed to standard values; for example, the metallicity was fixed to $Z=0.02$. Finally, the CEFF equation of state (Christensen-Dalsgaard \& Däppen 1992) and a $T(\tau)$ relation derived from a solar atmosphere model were used.

In theoretical computations, a standard upper boundary condition for eigenmodes is to impose in the uppermost layer the simple adiabatic evanescent solution for an isothermal atmosphere. In this case eigenmodes have frequencies below a cut-off frequency given by $\omega_{\text {cut }}=2 \pi v_{\text {cut }}=c / 2 H$, where $H$ is the density scale height. This is strictly speaking the cut-off frequency for radial oscillations, which is accurate for low-degree modes. To better fulfil the quasi-isothermal requirement, the boundary condition should be placed near the minimum temperature. For the Sun, this corresponds to an optical depth of about $\log \tau=-4$. Another advantage of using this sort of low optical depth is that at this position the oscillations are not too far from being adiabatic, at least compared to the photosphere. In our computations, we used this value as the uppermost point for all the models, but for some red giant stars we found that the maximum value of $\omega_{\text {cut }}(r)$ in the atmosphere can be located at higher optical depths; hence, we took that maximum value as representative 


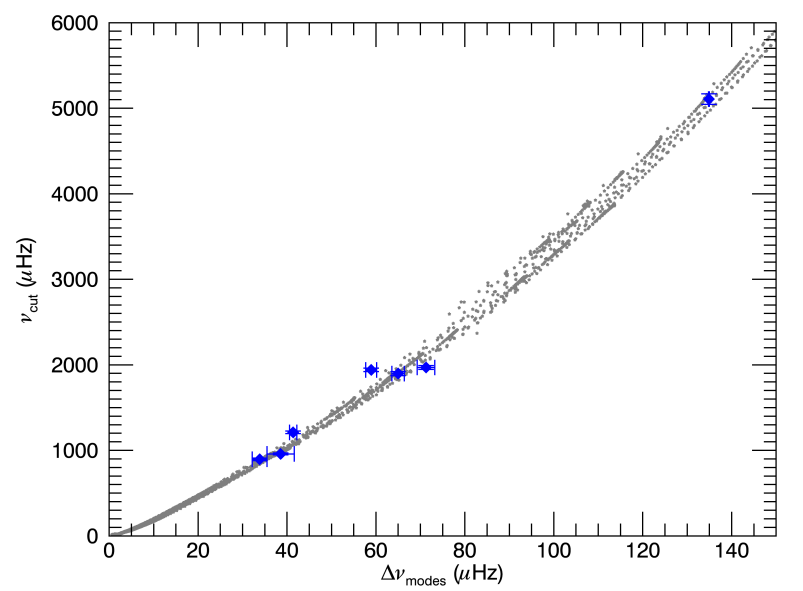

Fig. 7. $v_{\text {cut }}$ versus $\Delta v_{\text {modes }}$ for our set of theoretical models (grey dots). Blue points correspond to the observed stars, including the Sun (upper-right point).

of the observed $\omega_{\text {cut }}$. From an interpolation to the solar mass and radius (with $Y=0.25$ fixed), we obtained a value of $v_{\text {cut }}=$ $5125 \mu \mathrm{Hz}$ for the Sun from our set of models, which is in agreement with the observed value: $v_{\text {cut }}=5106.41 \pm 61.53 \mu \mathrm{Hz}$ (Jiménez 2006). If model S (Christensen-Dalsgaard et al. 1996) is considered, the differences are a little larger, about $4 \%$. It is important to remember that the solar $v_{\text {cut }}$ changes with the magnetic activity cycle (Jiménez et al. 2011); thus, some additional dispersion in the observed stellar values could be due to that effect.

For the large separation, the theoretical and observed values can be computed using the same approach. Specifically, we computed the large separation $\Delta v_{\text {modes }}$ from a polynomial fit of the form $\left(v-v_{0}\right) /\left(n-n_{0}\right)=\sum_{i} a_{i} P_{i}(x)$, where $n$ is the radial order and $v_{0}$ is the radial frequency closest to $v_{\max }$ with radial order $n_{0}$. For the models, $v_{0}$ is estimated assuming a linear relation to the cut-off frequency. The frequencies considered are those of the $\ell=0$ modes with radial orders $n=n_{0} \pm 4$. $P_{i}(x)$ is the Legendre polynomial of degree $i$, and $x$ is the frequency normalized to the interval $[-1,1]$. We used third order polynomial. From the Tasoul equation we expect that $a_{0} \equiv \Delta v_{\text {modes }} \simeq \Delta v$, whereas other terms mostly contain upper-layer information. We use only radial oscillations because, for evolved stars, the mixed character of some modes can introduce complications. For the Sun, we obtain $\Delta v_{\text {modes }}=134.9 \mu \mathrm{Hz}$ from the observations and $\Delta v_{\text {modes }}=136.0 \mu \mathrm{Hz}$ from model S.

Figure 7 shows $v_{\text {cut }}$ against the large separation $\Delta v_{\text {modes }}$ for our set of models and the observed stars. At first glance, there is rough agreement between the observed and theoretical calculations, but for the group of stars with $v_{\text {cut }} \sim 2000 \mu \mathrm{Hz}$, it seems hard to explain the dispersion in their $\Delta v_{\text {modes }}$ values.

We can proceed by taking the values of $T_{\text {eff }}$ into account. First, given the approximated scale relation for $v_{\text {cut }}$, we compute the residuals

$\delta_{\mathrm{c}}=1-\frac{v_{\mathrm{cut}}^{0}}{v_{\mathrm{cut}}} \frac{g \sqrt{\mu / T_{\mathrm{eff}}}}{\left(g \sqrt{\mu / T_{\mathrm{eff}}}\right)_{0}}$,

where $v_{\text {cut }}^{0}=5079.5 \mu \mathrm{Hz}$ is a reference cut-off frequency, corresponding to a model in our data set close to the Sun $\left(M=1 M_{\odot}\right.$, $\left.R=1.004 R_{\odot}, Y=0.25, T_{\mathrm{eff}}^{0}=5799.2 \mathrm{~K}\right)$. The $T_{\text {eff }}$ values are shown in Fig. 8. As seen in the figure, the differences can be as large as $\left|\delta_{\mathrm{c}}\right| \approx 10 \%$. In fact, the scale relation

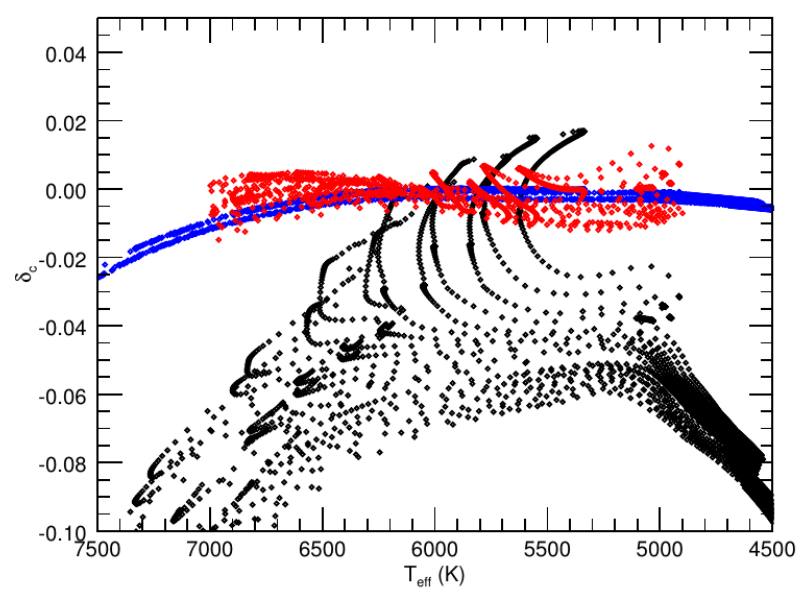

Fig. 8. Relative differences $\delta_{\mathrm{c}}$ as a function of $T_{\text {eff }}$ (black dots). Blue points are the same relative differences but considering the isothermal cut-off frequency $\omega_{\mathrm{a}}$ instead of $\omega_{\mathrm{c}}$. Red points are the residuals after correcting with the polynomial fit indicated in the text. The fit is limited to models with $T_{\text {eff }}<7000 \mathrm{~K}$ and $v_{\text {cut }}>500 \mu \mathrm{Hz}$.

$v_{\text {cut }} \propto g \sqrt{\mu / T_{\text {eff }}}$ is derived theoretically by approximating the density scale height, $H$, by the pressure scale height, $H_{\mathrm{p}}$, which is strictly valid in an isothermal atmosphere, and further by taking the equation of state of a mono-atomic ideal gas. In Fig. 8, the blue points correspond to the relative differences $\delta_{\mathrm{a}}$ obtained by replacing in Eq. (1) $\omega_{\text {cut }}=c / 2 H$ by $\omega_{\mathrm{a}}=c / 2 H_{p}$. Thus, the first condition introduces the highest departures from the scale relation, which was expected because in the range of $T_{\text {eff }}$ considered, the gas is mainly mono-atomic at the surface.

Nevertheless, as seen in Fig. $8, \delta_{\mathrm{c}}$ is mainly a function of $T_{\text {eff }}$. Hence, the scale relation can be improved if we subtract a polynomial fit from $\delta_{\mathrm{c}}$. We only considered models with $T_{\text {eff }}<7000 \mathrm{~K}$ and $v_{\text {cut }}>500 \mu \mathrm{Hz}$ in that fit because this range includes all the stars used in the present study. For a better fit we also consider the dependence of $\delta_{\mathrm{c}}$ on $v_{\text {cut }}$. In particular, the red points in Fig. 8 are obtained by replacing $v_{\text {cut }}$ in Eq. (1) by

$$
\begin{aligned}
v_{\text {cut }}^{*}= & v_{\text {cut }}\left[1-\left(0.0004-0.0057 x-1.0684 x^{2}+0.0543 y\right.\right. \\
& \left.\left.+0.1265 x y-0.7134 x^{2} y\right)\right] \frac{v_{\text {cut }}(\odot)}{v_{\text {cut }}^{*}(\odot)}
\end{aligned}
$$

where $x=T_{\text {eff }} / T_{\text {eff }}^{0}-1$ and $y=v_{\text {cut }} / v_{\text {cut }}^{0}-1$. The standard deviation for these residuals is $0.3 \%$. Hence, the modified cutoff frequencies, which we calibrated on the Sun, deviate by $1 \sigma=$ $0.3 \%$ from the scaling relation, at least for our set of models.

With a similar approach, we compute the deviation of $\Delta v_{\text {modes }}$ from its expected scale relation, namely,

$\delta_{\Delta}=1-\frac{\left(\Delta v_{\text {modes }}\right)^{0}}{\Delta v_{\text {modes }}} \sqrt{\frac{M / M_{0}}{\left(R / R_{0}\right)^{3}}}$,

where $\left(\Delta v_{\text {modes }}\right)^{0}$ is the value for our reference model, the same as the value used in $v_{\text {cut }}$. This quantity is shown in Fig. 9 for our set of models.

From Fig. 9 we can determine that the differences between the large separation computed from $p$-modes and the scaling relation can be as large as $5 \%$ for solar-like pulsators with $T_{\text {eff }}<$ $7000 \mathrm{~K}$. However, as shown in this figure, the differences $\delta_{\Delta}$ depend mainly on $T_{\text {eff }}$. This was previously noted by White et al. (2011) and allows for a correction to $\Delta v_{\text {modes }}$ to obtain a better 
Table 3. Stellar parameters II.

\begin{tabular}{lccccc}
\hline \hline KIC & $\log g_{\text {sp }}$ & $\log g_{v_{\max }}$ & $\log g_{v_{\text {cut }}}$ & $\Delta v_{\text {modes }}$ & $\bar{\rho}$ \\
\hline 3424541 & $3.50 \pm 0.08$ & $3.84 \pm 0.03$ & $3.86 \pm 0.02$ & $38.8 \pm 1.2$ & $0.116 \pm 0.007$ \\
7799349 & $3.71 \pm 0.08$ & $3.67 \pm 0.02$ & $3.68 \pm 0.02$ & $33.3 \pm 0.2$ & $0.085 \pm 0.001$ \\
7940546 & $4.11 \pm 0.08$ & $4.01 \pm 0.02$ & $4.06 \pm 0.02$ & $58.9 \pm 0.4$ & $0.270 \pm 0.004$ \\
9812850 & $4.16 \pm 0.08$ & $4.06 \pm 0.03$ & $4.06 \pm 0.02$ & $64.4 \pm 0.5$ & $0.327 \pm 0.005$ \\
11244118 & $4.23 \pm 0.08$ & $4.09 \pm 0.03$ & $4.04 \pm 0.02$ & $71.5 \pm 0.5$ & $0.388 \pm 0.005$ \\
11717120 & $3.80 \pm 0.08$ & $3.69 \pm 0.02$ & $3.71 \pm 0.02$ & $37.7 \pm 0.3$ & $0.109 \pm 0.002$ \\
\hline
\end{tabular}

Notes. Columns: values of $\log g$ derived from spectroscopy, $v_{\max }$ and $v_{\text {cut }}$ for each star in our sample. The mean density $\bar{\rho}$ is in $\mathrm{g} / \mathrm{cm}^{3}$ and the large separation $\Delta v_{\text {modes }}$ in $\mu \mathrm{Hz}$. See text for details.

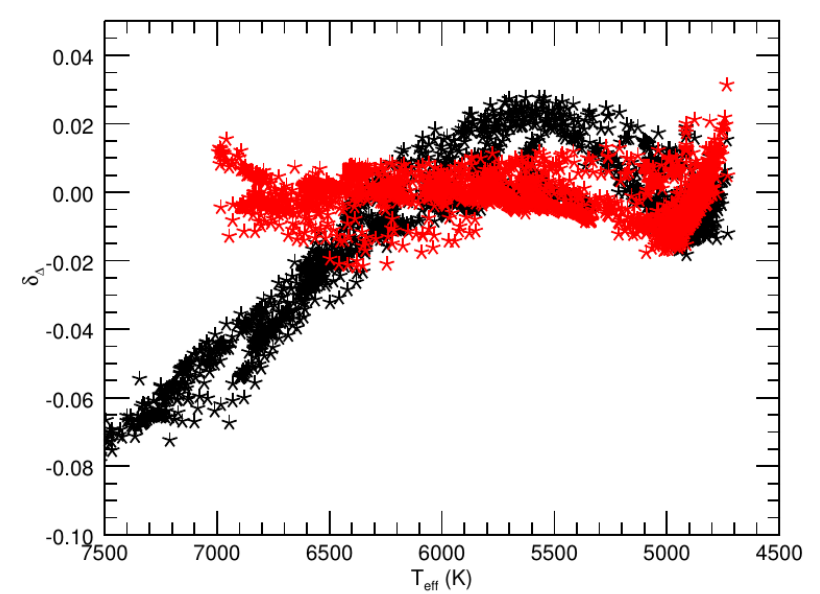

Fig. 9. Relative differences $\delta_{\Delta}$ versus $T_{\text {eff }}$ for the set of models indicated in the text (black dots). The red points are the residuals after the polynomial fit. This fit is limited to models with $T_{\text {eff }}<7000 \mathrm{~K}$ and $\Delta v>10 \mu \mathrm{Hz}$.

estimate of the mean density. Here we use a fit similar to that considered for $v_{\text {cut }}$. As in the previous case, we have considered only those models with $T_{\text {eff }}<7000 \mathrm{~K}$ and $\Delta v_{\text {modes }}>10 \mu \mathrm{Hz}$, which include all the stars used in the present work. The red points in Fig. 9 correspond to the residuals obtained after replacing $\Delta v_{\text {modes }}$ in Eq. (3) by

$$
\begin{aligned}
\Delta v_{\text {modes }}^{*} & =\Delta v_{\text {modes }}\left[1-\left(0.0023-0.1123 x-0.7309 x^{2}\right.\right. \\
& \left.\left.-0.0299 z-0.0067 x z+1.2461 x^{2} z\right)\right] \frac{\Delta v_{\text {modes }}(\odot)}{\left(\Delta v_{\text {modes }}\right)^{*}(\odot)}
\end{aligned}
$$

where $x=T_{\text {eff }} / T_{\text {eff }}^{0}-1$ and $z=\Delta v_{\text {modes }} /\left(\Delta v_{\text {modes }}\right)^{0}-1$ with $\left(\Delta v_{\text {modes }}\right)^{0}=135.4 \mu \mathrm{Hz}$. The standard deviation for these residuals is $0.6 \%$. Given the differences between the observed and theoretical values for the Sun, we calibrated Eq. (4) to the observed solar value.

Figure 10 shows $v_{\text {cut }}^{*}$ against $\Delta v_{\text {modes }}^{*}$ for our set of models (black points) and the observed stars including the Sun (blue points). Whereas the scattering in the theoretical values are substantially reduced, the observational values are not. Hence, if the error estimates are correct, we must conclude that the observational cut-off frequencies do not completely agree with the theoretical estimates. Moreover, the red points in Fig. 10 are proportional to the observed values of the frequency of maximum amplitude scaled to the Sun, $\left(v_{\text {cut }} / v_{\max }\right)_{\odot} v_{\max }$. For our limited set of stars, $v_{\max }$ follows the scaling relation better than $v_{\text {cut }}^{*}$. This is a

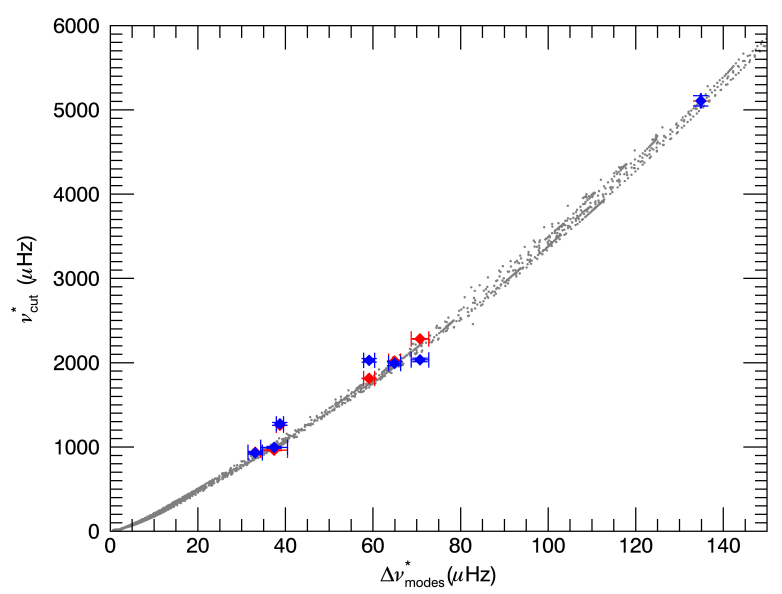

Fig. 10. Effective $v_{\text {cut }}^{*}$ versus the effective $\Delta v_{\text {modes }}^{*}$ for our set of models (grey dots) and for the observed stars (blue dots). Red points are observed values of the frequencies of maximum amplitude properly scaled, $\left(v_{\text {cut }} / \nu_{\max }\right)_{\odot} v_{\max }$.

little surprising because, as noted before, theoretically, we would expect the opposite, even more so when the effective $v_{\text {cut }}^{*}$ is used.

This result can be understood in terms of the inferred surface gravity since we can use either $v_{\max }$ or $v_{\text {cut }}$ to estimate $\log g$. The results are summarized in Table 3 and compared to the spectroscopic $\log g_{\mathrm{sp}}$. To estimate the errors in $\log g_{v_{\max }}$ we only considered the observational errors in $v_{\max }$ and $T_{\text {eff }}$, while for $\log g_{v_{\text {cut }}}$ we have included the $1 \sigma$ value of $0.3 \%$ found in the scaling relation plus an error in $\mu_{\mathrm{s}}$ derived from assuming an unknown composition with standard stellar values. In particular, we have taken ranges of $Y=[0.24,0.28]$ and $Z=[0.01,0.03]$ for the helium abundance and the metallicity, respectively, and thus estimated the error in the mean molecular weight $\mu_{\mathrm{s}}$ by some $5 \%$. As expected, the values obtained from $\log g_{v_{\max }}$ are quite similar to those reported by Bruntt et al. (2012) because they are based on the same global parameters with very similar values. The values derived from $v_{\max }$ and $v_{\text {cut }}$ are also much closer to each other than those derived spectroscopically, which, as mentioned before, have large observational uncertainties.

\section{The HIP region}

\subsection{Observations}

Using Sun-as-a-star observations from the GOLF instrument on board SoHO, García et al. (1998) uncovered the existence of a 
sinusoidal pattern of peaks above $v_{\text {cut }}$, as the result of the interference between two components of a travel wave generated on the front side of the Sun with a frequency $v \geq v_{\text {cut }}$, where the inward component returns to the visible side after a partial reflection on the far side of the Sun (see Fig. 3 in García et al. 1998). Therefore, the frequency spacing of $\sim 70 \mu \mathrm{Hz}$ found in the Sun corresponded to the time delay between the direct emitted wave and that coming from the back of the Sun, corresponding to waves behaving like low-degree modes, i.e. a delay of four times the acoustic radius of the Sun $(\sim 3600 \mathrm{~s})$. This value is roughly half of the large frequency spacing of the star, which we call $\Delta v_{1}$. García et al. (1998) also speculated that, above a given frequency, a second pattern should become visible with a double frequency spacing (close to the large frequency separation), $\Delta v_{2} \sim 140 \mu \mathrm{Hz}$. This pattern (for a theoretical description, see Kumar 1993), which is usually visible in imaged instruments (Duvall et al. 1991), corresponds to the interference between outward emitted waves and the inward components, which arrive at the visible side of the Sun after the refraction at the inner turning point (non-radial waves).

Two sine waves are fitted above the cut-off frequency to obtain a global estimate of the frequency of the interference patterns in the HIP region of our sample of stars. The amplitudes and frequencies of both sine waves are summarized in Table 2. We also reanalysed the GOLF and VIRGO data, following the same procedure using at a higher frequency range of between 7 and $8 \mathrm{mHz}$, compared to the original analyses performed by García et al. (1998) and Jiménez (2006). With this approach, we have been able to obtain the second periodicity at $\sim 140 \mu \mathrm{Hz}$ (see Table 2).

We tried fitting various functions and opted for the simplest one. Indeed, the amplitude of the interference patterns decreases with frequency in a manner that is close to an exponential decrease. The additional parameters required give more unstable fits with a heavy dependence on the guess parameters. We preferred this approach because we obtained the same qualitative results leading to the same classification of stars. In future studies, we will look for a better function for the fit, i.e. one more suited to the observations, in a larger set of stars. We are already working in this direction but this investigation is beyond of the scope of this paper.

According to the fitted amplitudes, we can classify the stars into two groups: those in which the two amplitudes of the sine waves, $A_{1}$ and $A_{2}$, are similar (KIC 7940546, KIC 9812850, KIC 11244118), and those in which $A_{2}$ is much larger than $A_{1}$ (KIC 3424541, KIC 7799349, KIC 11717120). An example of each group of stars is given in Fig. 11 for stars KIC 7940546 and KIC 11717120. The error bars of the fitted amplitudes are large because the actual amplitudes of the interference patterns decrease in a quasi-exponential way that has not been taken into account here. We favoured the fits with constant amplitudes to avoid adding more unknown parameters to the fit.

The stars of the first group have larger $\Delta v_{\text {modes }}$ than the other three stars, which implies a correlation with the evolutionary state of the stars. Starting with the Sun on the main sequence, the HIP pattern is dominated by interference waves partially reflected at the back of the Sun (for the solar case $A_{1}$ is much bigger than $A_{2}$ ). When the stars evolve, the HIP pattern due to the interference of direct with refracted waves in the stellar interior is more and more visible and becomes dominant for evolved RGB stars with $\Delta v_{\text {modes }}$ below $\sim 40 \mu \mathrm{Hz}$.
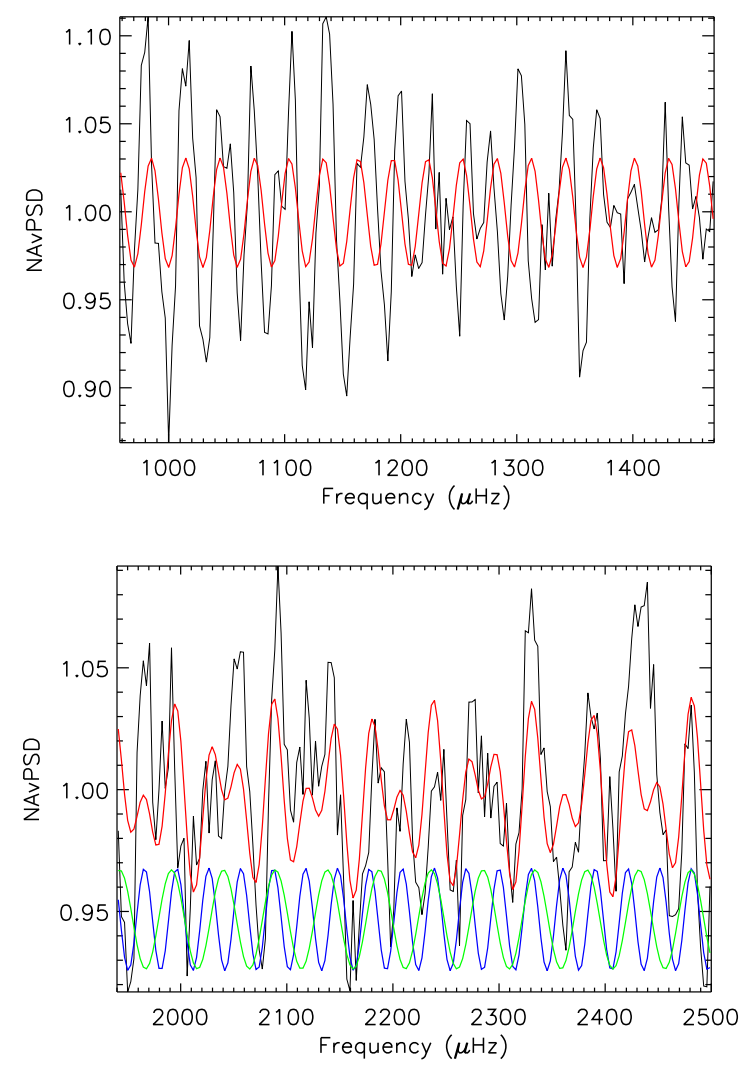

Fig. 11. HIPs region of KIC 11717120 (top), where the HIP pattern is dominated by the sine wave (red line), owing to the interference between direct and refracted waves; and the region of KIC 7940546 (bottom), where the two HIP patterns can be determined. The blue and green lines in the bottom panel correspond to the two fitted sine waves with frequencies $\Delta v_{1}$ and $\Delta v_{2}$, respectively. We shifted the green and blue lines by 0.05 for clarity. The red line is the actual fit.

\subsection{HIPs and stellar evolution}

To reproduce the periodic signals expected from the pseudomode spectrum theoretically, we follow the interpretation given by Kumar (1993) and García et al. (1998) and assume that waves are excited isotropically at a point very close to the photosphere. The observed spectrum of the pseudo-modes is then interpreted as an interference pattern between outgoing and ingoing components, possibly including successive surface reflections. In what follows, we summarize the basic concepts and apply them to stars at different evolutionary stages.

We start with a gravito-acoustic ray theory approximation with the following dispersion relation (see e.g. Gough 1986, 1993):

$k^{2}=k_{\mathrm{r}}^{2}+k_{\mathrm{h}}^{2}=\frac{1}{c^{2}}\left(\omega^{2}-\omega_{c}^{2}\right)+\frac{N^{2}}{\omega^{2}} k_{\mathrm{h}}^{2}$,

where $k_{\mathrm{r}}$ is the radial component of the wave number, $k_{\mathrm{h}}^{2}=$ $L^{2} / r^{2}, L=l(l+1)$ the horizontal component, $N$ is the buoyancy frequency, $\omega_{c}$ is a generalized cut-off frequency given by

$\omega_{c}^{2}=\frac{c^{2}}{4 H^{2}}\left(1-2 \frac{\mathrm{d} H}{\mathrm{~d} r}\right)$,

and $H$ is the density scale height. In the ray approximation, the turning points are given by $k_{\mathrm{r}}=0$, whereas the ray path is determined by the group velocity. For the spherically symmetric 
case, the rays are contained in a plane with a path given by $\mathrm{d} \theta / \mathrm{d} r=v_{\theta} /\left(r v_{r}\right)$. Here, $r$ and $\theta$ are the usual polar coordinates and the group velocity $\boldsymbol{v}_{\mathrm{g}}=\left(v_{r}, v_{\theta}\right)=\left(\partial \omega / \partial k_{r}, \partial \omega / \partial k_{\mathrm{h}}\right)$ has the following components:

$\boldsymbol{v}_{\mathrm{g}}=\left(\frac{k_{r} \omega^{3} c^{2}}{\omega^{4}-k_{\mathrm{h}}^{2} c^{2} N^{2}}, k_{\mathrm{h}} \omega c^{2}\left[\frac{\omega^{2}-N^{2}}{\omega^{4}-k_{\mathrm{h}}^{2} c^{2} N^{2}}\right]\right)$.

According to the ray theory, the general solution of the wave equation can be expressed as a superposition of rays of the form $\psi_{\omega}(r, t)=A_{\omega}(r) \exp \left[\mathrm{i}\left(\omega t \pm \int_{S} k \mathrm{~d} s\right)\right]$, where the two signs corresponds to outgoing and ingoing waves and the integral is computed over the ray path $s$, including additional reflections where appropriate. For every reflection, a constant phase shift must be introduced. In particular, for a ray travelling from an inner turning point $r_{1}$ to an outer turning point $r_{2}$, this integral can be expressed as

$\frac{\omega}{2 \Delta v_{\omega, \ell}} \equiv \int_{s} k \mathrm{~d} s=\int_{r_{1}}^{r_{2}} k \frac{v_{\mathrm{g}}}{v_{r}} \mathrm{~d} r-\pi / 4$

For low-degree acoustic waves $\omega /\left(2 \Delta v_{\omega, \ell}\right) \simeq \omega \int_{0}^{R}(1 / c) \mathrm{d} r-(1+$ $\ell) \pi / 2$, that is, $\Delta v_{\omega, \ell} \approx \Delta v$.

Considering a wave excited very close to the surface with an outgoing component of amplitude $A_{\omega \ell}$ and an ingoing component that emerges at the surface with an amplitude $A_{\omega \ell}^{\prime}$ after its first internal traversal, and with an amplitude of $A_{\omega, \ell}^{\prime} R(\omega)$ after a subsequent partial surface reflection on the back side of the star, the contribution to the amplitude spectrum can be expressed as

$\sum_{\omega, \ell}\left(V_{\omega, \ell} A_{\omega, \ell}+A_{\omega, \ell}^{\prime} \mathrm{e}^{\mathrm{i} \delta}\left[V_{\omega, \ell}^{\prime} \mathrm{e}^{-\mathrm{i} \omega / \Delta v_{\omega, \ell}}+V_{\omega, \ell}^{\prime \prime} R(\omega) \mathrm{e}^{-2 \mathrm{i} \omega / \Delta v_{\omega, \ell}}\right]\right)$,

where $V_{\omega, \ell}, V_{\omega, \ell}^{\prime}$ and $V_{\omega, \ell}^{\prime \prime}$ are visibility factors and $\delta$ is a phase constant that depends on the type of radiation emitted. The reflection coefficient $R$ is expected to be a monotonically decreasing function of frequency (for further details see Kumar 1993). We also assume that, for stochastically excited waves, the amplitudes and the visibility factors are a smooth function of frequency. Hence, the amplitude spectrum is modulated with the periodic functions $\cos \left(\omega / \Delta v_{\omega, \ell}\right)$ and $\cos \left(2 \omega / \Delta v_{\omega, \ell}\right)$. Although in principle different values of $\Delta v_{\omega, \ell}$ can be expected in different frequency ranges and for different degrees $\ell$, their differences are too weak, and in the present study we fitted the data to just two periodic components, $\Delta v_{2}=\Delta v_{\omega, \ell}$ and $\Delta v_{1}=\Delta v_{\omega, \ell} / 2$. In addition, in some stars one of the signals could be masked by low-visibility factors or low reflection coefficients.

To illustrate the problem, we considered a $1.1 M_{\odot}$ evolution sequence and plotted rays for two typical frequencies in the pseudo-mode range and three evolutionary stages in Fig. 12. Top panels are for frequencies $\omega=1.1 \omega_{\mathrm{a}}$ while bottom panels are for $\omega=1.5 \omega_{\mathrm{a}}$. On the other hand, the star evolves from left (zero age main sequence, ZAMS) to right (red giant branch, RGB). For clarity, surface reflections have been omitted.

For the model close to the ZAMS (leftmost panels), the inward rays emerge at the surface making angles between $160^{\circ}$ and $180^{\circ}$ with the outward component. In this case, the outward and inward components of a given wave can only be simultaneously visible if they lie too close to the limb. Hence, their signal corresponding to $\Delta v_{2} \simeq \Delta v$ is highly attenuated in the power spectrum. In contrast, after one surface reflection the angle between the outward and inward components lies in the range $0^{\circ}$ to $35^{\circ}$. This interference would give the $\Delta v_{1} \simeq \Delta v / 2$ separation
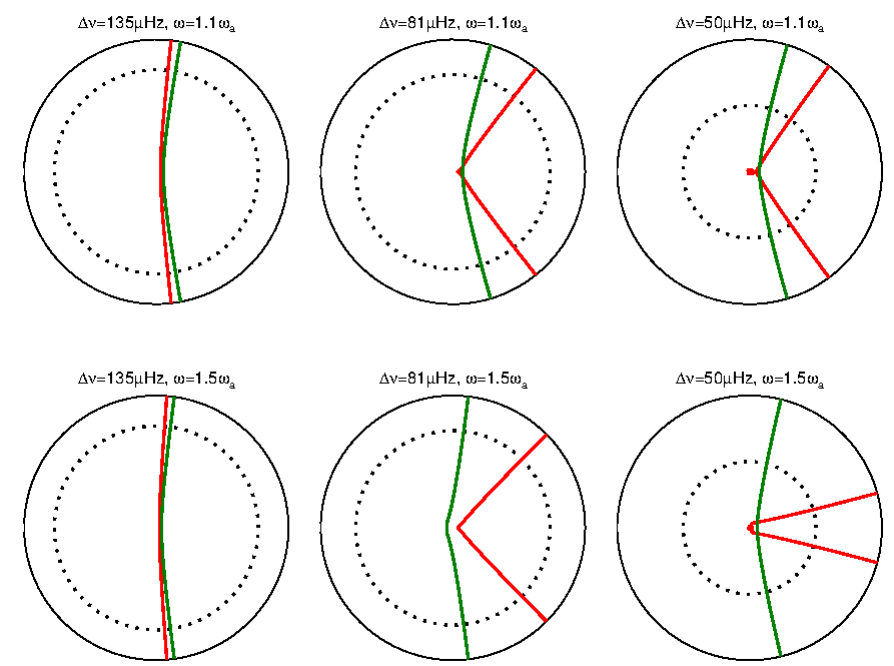

Fig. 12. Ray path for waves with angular degrees $\ell=1$ (red) and $\ell=2$ (green) for a $1.1 M_{\odot}$ model evolution sequence. For clarity, surface reflections have been omitted. The upper part shows rays with a frequency a $10 \%$ greater than the acoustic cut-off frequency $\omega_{a}$, while the bottom panels corresponds to rays with a frequency $50 \%$ greater than $\omega_{\mathrm{a}}$. The large separation indicated is the integral $\left(2 \int_{0}^{R} 1 / c\right)^{-1}$ and can be used to identified the models in Fig. 13. The leftmost panels correspond to a model near the ZAMS, the middle panels indicate a model near the TAMS, and the rightmost panels indicate a model in the RGB. The dotted circles indicate the base of the convection zone.

and, hence, if it had the same intrinsic amplitude as the former, the $\Delta v_{1}$ separation would be easier to observe. In this case, however, the inward rays are only partially reflected on the far side (e.g. Kumar 1993) and hence the attenuation factor, corresponding to $R(\omega)$ in Eq. (9), is high. Since the actual observations show that in the Sun the dominant separation in the HIP pattern is $\Delta v_{1}$ (García et al. 1998), we can use this case as a qualitative reference between the two competing factors. For model S, we obtain $\Delta v_{1}=72 \mu \mathrm{Hz}$ with our fit, for which a frequency range $\sim[5200$, $6600] \mu \mathrm{Hz}$ was used. This result is in agreement with the observational value of $70.46 \pm 2 \mu \mathrm{Hz}$ found by García et al. (1998). As mentioned in section 4.1, we reanalysed the solar data (GOLF and VIRGO Sun photometers) and obtained $\Delta \nu_{1} \sim 70 \mu \mathrm{Hz}$ (see Table 2), in agreement with previous values given in the literature. At higher frequencies we refitted the data with two sinusoidal components and found $\Delta v_{2} \sim 140 \mu \mathrm{Hz}$ (Table 2). This value is in agreement with the theoretical prediction already described by Garcia et al. (1998) if we assume that the photosphere is the only source of partial wave reflection. However, in this case the amplitude is much smaller because only waves close to the limb contribute to this interference pattern.

As the stars evolve, the angle between the outward ray and the first surface appearance of the inward refracted ray becomes smaller for the $\ell=1$ pseudo-modes. This is clearly apparent in Fig. 12. Hence, the $\Delta v_{2} \simeq \Delta v$ interference pattern becomes easier to observe (higher observed $A_{2}$ amplitudes). This phenomenon is due to the transition between acoustic rays smoothly bending through the stellar interior, and waves where the density scale height in the stellar inner structure becomes close to their wavelength and the ray behaviour at the inner turning point is closer to a two-layer reflection. Finally, in the most evolved model in Fig. 12 (upper and bottom right panels) the $\ell=1$ waves have a gravity character close to the centre that bends the ray into a loop. 


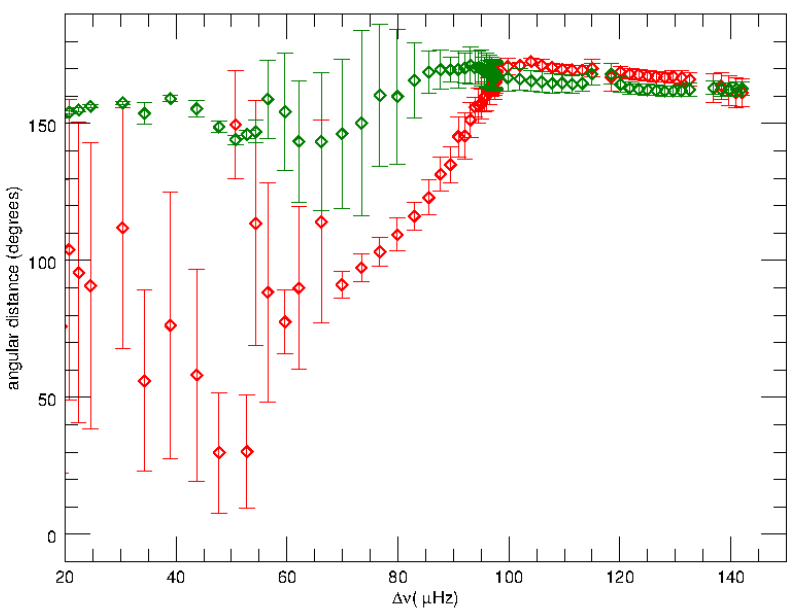

Fig. 13. Angular distance between inward and outward waves against $\Delta v$ for a $1.1 M_{\odot}$ evolution sequence. Frequencies between the acoustic cut-off frequency at the surface and about 50\% greater than $\omega_{\mathrm{a}}$ are considered. For every model in the sequence, frequency-averaged values of the angular distance and $1 \sigma$ dispersions are calculated separately for $\ell=1$ (red points) and $\ell=2$ (green points) waves. An angle of $180^{\circ}$ means no inner reflection.

We used Eq. (9) to reproduce the observed power spectrum schematically, and considered ray traces for a continuum spectrum of waves with frequencies between the acoustic cut-off frequency at the surface and about 1.5 times that value. This frequency interval spans approximately the observed pseudo-mode frequency range. Since we are only interested in reproducing the periodicities of the pseudo-mode spectra, constant amplitudes were considered as well as visibility factors either constant or proportional to $\cos \Theta / 2$, where $\Theta$, is the angular distance between the outward and inward waves. We determine the periodic signal in the spectrum with a non-linear fit to the above equation.

Figure 13 shows the angular distance between the inward and outward components before any surface reflection against the large separation $\Delta v$ for models in a $1.1 M_{\odot}, Y=0.28$ evolution sequence with the code and physics indicated in Sect. 3.2. Here, mean values of the angular distance are computed by averaging those of the ray traces in the frequency range indicated above. Pseudo-modes with $\ell=1$ and 2 are averaged separately. Since no surface reflection is considered, these waves form the $\Delta v_{2}$ pattern.

Looking at the behaviour of the $\ell=1$ pseudo-modes in Fig. 13 (red points), we may conclude that for $M=1.1 M_{\odot}$ stars that evolve to a $\Delta v$ below some given threshold between $90 \mu \mathrm{Hz}$ and $60 \mu \mathrm{Hz}$ (corresponding to evolved main-sequence stars as that shown in the middle panel of Fig. 12), the $\Delta v_{2}$ pattern should become visible. Indeed, a smaller angular distance means more disc-centred interferences favouring a higher $A_{2}$. The critical $\Delta v$ slightly changes with mass, which is lower for higher masses. In any case, all the stars in Table 2, except the Sun, are evolved to a point where the pattern with $\Delta v_{2}$ could be easily observed, as is in fact the case.

Regarding the $\ell=2$ pseudo-modes, it can be seen in Fig. 13 that the angle distance always remains above some $150^{\circ}$ and hence this degree only contributes to the $\Delta v_{1}$ period in the pseudo-mode spectrum. The higher dispersion in the angular distance for models with a large separation between $\Delta v=60$ and $90 \mu \mathrm{Hz}$ (evolved main-sequence stars and subgiants), clearly visible in Fig. 13, deserves a comment. According to the dispersion relation we used, for models evolved near the Terminal Age Main Sequence (TAMS) the characteristic frequencies of the $\ell=2$ waves become complex in a small radius interval above the core, thus increasing the resonant cavity of the pseudo-modes within a limited frequency range. An example of the ray path of these kinds of waves is shown in the middle of the bottom panel of Fig. 12 (green line). Here, the ray trace close to the centre is not of the acoustic type and the emerging ray is consequently scattered. Althought these results may be questionable in terms of the validity of the approximation used here, they do not have any observable consequences.

For the three most evolved stars in our data set the interference pattern coming from waves reflected back at the surface with period $\Delta v_{1}$ is not observed. The fact that for evolved stars the signal from the $\ell=1$ pseudo-modes do not suffer any attenuation from surface reflections may explain why for stars evolved to the RGB the $\Delta v_{1}$ signal becomes completely masked. However, it is interesting to note the another, possibly superimposed, cause for this circumstance. In principle, one might expect that radial waves propagates all the way from the surface to the centre and hence contributes to the signal only once reflected back, hence, with a period $\Delta v_{1}$. However there is a point in the evolution where the cut-off frequency in the core rises above the typical pseudo-mode frequencies, in which case the ray theory introduces an inner reflection. Although a plano-parallel approximation is questionable in terms of the wavelength-radius relation when we are too close to the centre, this approximation allows us to estimate the transmission coefficient $T$, as in the one-dimensional problem ${ }^{1}$. If the equation $T=1 /\left(1+\mathrm{e}^{2 K}\right)$ is used with $K=2 \int_{0}^{r_{1}} \sqrt{\left(\omega_{c}^{2}-\omega^{2}\right) / c^{2}}, r_{1}$ being the point where $k_{r}=0$, it happens that, for red giant stars, radial waves in the observed frequency range of the pseudo-mode spectrum are mostly reflected at the core edge. Thus, at this stage, radial pseudomodes also contribute to the $\Delta v_{2}$ signal and, hence, the $\Delta v_{1}$ period would hardly be observed.

We now discuss the relation between the large separation obtained from the eigenmodes, $\Delta v_{\text {modes }}$, and those corresponding to the pseudo-modes, $\Delta v_{1}$ and $\Delta v_{2}$. First, we verified that for waves with $\ell=0$ the phase travel time is always close to the asymptotic acoustic value, $\int k \mathrm{~d} l \simeq \int_{0}^{R} 1 / c \mathrm{~d} r$. Hence, when these acoustic waves contribute to the HIPs after a surface reflection they give $\Delta v_{1} \simeq \Delta v_{\text {modes }} / 2$. In addition, most of the $\ell=2$ and, in some stars, $\ell=1$ travelling waves have similar acoustic characteristics, which also contributed to the same interference period. This is in agreement with Table 2, where both frequency separations match within the errors.

The case of $\Delta v_{2}$ is different. The grey points in Fig. 14 are the periods of the interference pattern computed according to Eq. (9) for travelling waves and no surface reflection. A wide range of masses and evolutionary stages from main sequence to the base of the red-giant branch were included. The red points are the observed $\Delta v_{2}$ values while blue points are $2 \Delta v_{1}$ when observed, including the Sun. The black line corresponds to $\Delta v_{\text {modes }}=\Delta v_{2}$. From Fig. 14 we can see that, for $\Delta v_{\text {modes }}=60-80 \mu \mathrm{Hz}$, there are models with $\Delta v_{2}<\Delta v_{\text {modes}}$. In fact, they correspond to evolved stars up to the end of the main sequence or the subgiant phase, as it is the case for our sample of stars. Although the order of magnitude of the phase delay found for our models is similar to

1 For radial oscillations, the full adiabatic equations are of second order and, hence, a WKB analysis can be obtained without the approximations assumed in the dispersion relation Eq. (5). The qualitative results given in this paragraph rely solely on the assumption of a one-dimensional problem. 


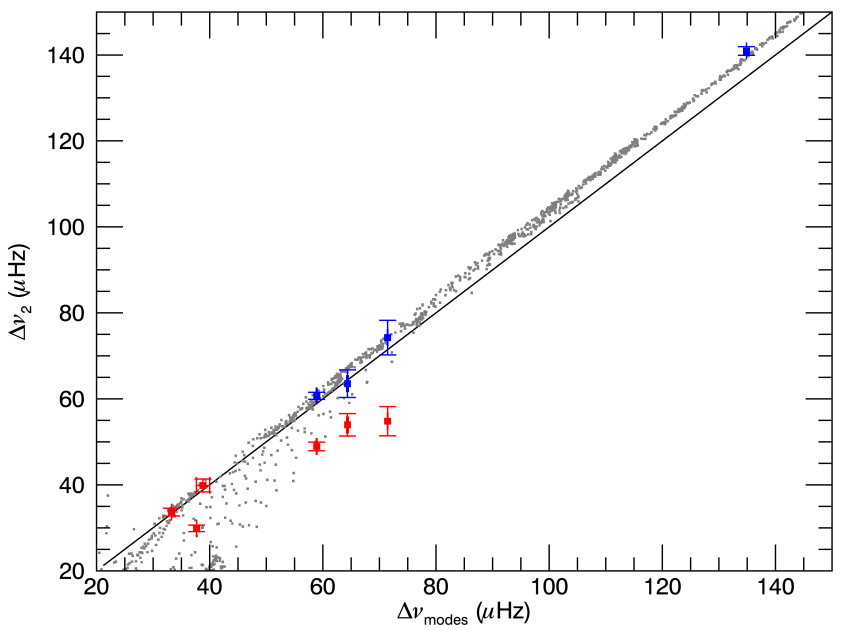

Fig. 14. Grey points are periods of the pseudo-mode interference pattern for evolution sequences with masses between $0.9 M_{\odot}$ and $2 M_{\odot}$ For the sake of clarity, only waves with no surface reflections are shown. Red points correspond to the observed $\Delta v_{2}$ values and blue points correspond to $2 \Delta v_{1}$ when observed. The black line corresponds to $\Delta v_{\text {modes }}=\Delta v_{2}$.

the observed one for this type of star, it is also apparent from the figure that KIC $1124411\left(\Delta \nu_{\text {modes }} \sim 70 \mu \mathrm{Hz}\right)$ has a large separation, which is too high for this delay to appear. We obtain a mass of $\sim 1 M_{\odot}$ either by applying the scaling relations to $v_{\max }$ and $\Delta v_{\text {modes }}$ via an isochrone fit. The upper right corner of Fig. 12 is representative of this star. Hence we expect to observe the signal $\Delta v_{2}$ from waves reflected in the interior but our computations do not show any significant delay in the phase of the $\ell=1$ compared to the radial oscillations. Further work is need here; in particular, the asymptotic theory that we have used could be inadequate for these kinds of details.

For stars with the lower $\Delta v_{\text {modes }}$, two of them have $\Delta v_{2} \simeq$ $\Delta v_{\text {modes }}$ and one star (KIC 11717120) has $\Delta v_{2}<\Delta v_{\text {modes }}$. With our isochrones fitting, we find that the latter is at the base of the RGB as well as KIC 7799349, thus we have two different results for stars with very similar parameters. With our simple simulation for models at this evolutionary stage we have both signals since, as noted above, radial oscillations are partially reflected at the core edge and, for these stars, $\Delta v_{2} \simeq \Delta v_{\text {modes }}$. The observed period is a weighted average, but for a better comparison with the observations proper amplitudes need to be computed.

\section{Conclusions}

We measured the acoustic cut-off frequency and characteristics of the HIPs in six stars with solar-like pulsations observed by the Kepler mission. A comparison with the observed $v_{\max }$ shows a linear trend with all stars lying in $\sim 2 \sigma$. As a result, the values of $\log g$ derived from $v_{\text {cut }}$ agree to within the same accuracy with those derived from $v_{\max }$, but are substantially different in some cases from the values derived from spectroscopic fits (see Table 3). When comparing the stars with the models in the $v_{\text {cut }}-\Delta v$ plane, we found a departure in $v_{\text {cut }}$ from the expected values. It is possible to calculate, with theoretical information, a measurable function $v_{\text {cut }}^{*}=f\left(T_{\text {eff }}, v_{\text {cut }}\right)$ that scales as $g / \sqrt{T_{\text {eff }}}$ with no more than $0.3 \%$ deviation (for our set of models representative of our star sample and the Sun), however, we find that the observational $v_{\text {cut }}^{*}$ does not follow this relation so accurately.
Rather surprisingly, we found that the frequency of maximum power, $v_{\max }$, follows the linear relation within errors. Hence, for the evolved stars considered in the present study it must be concluded that $v_{\max }$ gives an even better estimate of $\log g$ than $v_{\text {cut }}$ with the current uncertainties.

Given the observed characteristics of the HIPs, our set of six stars can be divided into two groups. In three stars: KIC 3424541, KIC 7799349, and KIC 11717120, the only visible pattern is that due to the interference with inner refracted waves in the visible disc of the star and not close to the limb. In the other three stars (KIC 7940546, KIC 9812850, and KIC 11244118), the pattern is because partial reflection of the inward waves at the back of the star is also detected. This different behaviour is related to the wide spacing of the star, which reveals a dependence on the evolutionary stage.

When present, the period $\Delta v_{1}$, which corresponds to the interference of outward waves with their inward counterpart once reflected on the far side, always agrees with half the large separation, although, like the Sun, their values are a little higher. This result is also found theoretically, and the large separation derived from the pseudo-modes is closer to $\int \mathrm{d} r / c$. However, the period $\Delta v_{2}$ corresponding to waves refracted in the inner part of the star can be substantially different from the large frequency separation. We interpret this by claiming the presence of waves of a mixed nature. For these stars, the phase $\int k \mathrm{~d} l$ is smaller compared to the radial acoustic case. Although a detailed analysis is beyond the scope of the present study, simple ray theory calculations reveal that this kind of a phenomenon is expected with the same order of magnitude.

The pseudo-mode spectrum reveals information from the surface properties of the stars through $v_{\text {cut }}$ and from the interior, at least in the cases where the period $\Delta v_{2}$ is lower than the large separation derived from the eigenmodes. We are aware that the interference periods derived observationally are power-weighted averages and for a proper comparison with theoretical expectation some work along these lines should be addressed. This might be accomplished by computing transmission coefficients so that more realistic theoretical simulations of the interference phenomenon can be performed.

Acknowledgements. The authors of this paper thank Dr. J. Ballot, Dr. G. R. Davies, and P. L. Pallé for useful comments and discussions, as well as the entire Kepler team, without whom these results would not be possible. Funding for this Discovery mission is provided by NASA Science Mission Directorate. This research was supported in part by the Spanish National Research Plan under project AYA2010-17803. This research was supported in part by the National Science Foundation under Grant No. NSF PHY05-51164. R.A.G. has received funding from the European Community Seventh Framework Program (FP7/2007-2013) under grant agreement No. 269194 (IRSES/ASK), from the ANR (Agence Nationale de la Recherche, France) program IDEE (No. ANR-12BS05-0008) "Interaction Des Étoiles et des Exoplanètes", and from the CNES. S.M. acknowledges the support of the NASA grant NNX12AE17G.

\section{References}

Bedding, T. R., \& Kjeldsen, H. 2003, PASA, 20, 203

Borucki, W. J., Koch, D., Basri, G., et al. 2010, Science, 327, 977

Brown, T. M., Latham, D. W., Everett, M. E., \& Esquerdo, G. A. 2011, AJ, 142, 112

Bruntt, H., Basu, S., Smalley, B., et al. 2012, MNRAS, 423, 122

Chaplin, W. J., Houdek, G., Appourchaux, T., et al. 2008, A\&A, 485, 813

Christensen-Dalsgaard, J. 2008, Ap\&SS, 316, 13

Christensen-Dalsgaard, J., \& Däppen, W. 1992, A\&ARv, 4, 267

Christensen-Dalsgaard, J., Dappen, W., Ajukov, S. V., et al. 1996, Science, 272, 1286

Domingo, V., Fleck, B., \& Poland, A. I. 1995, Sol. Phys., 162, 1 
A. Jiménez et al.: Acoustic cut-off frequency and HIPs with Kepler

Duvall, Jr., T. L., Harvey, J. W., Jefferies, S. M., \& Pomerantz, M. A. 1991, ApJ, 373, 308

Fröhlich, C., Romero, J., Roth, H., et al. 1995, Sol. Phys., 162, 101

Gabriel, A. H., Grec, G., Charra, J., et al. 1995, Sol. Phys., 162, 61

García, R. A., Pallé, P. L., Turck-Chièze, S., et al. 1998, ApJ, 504, L51

García, R. A., Hekker, S., Stello, D., et al. 2011, MNRAS, 414, L6

García, R. A., Mathur, S., Pires, S., et al. 2014, A\&A, 568, A10

Gilliland, R. L., Jenkins, J. M., Borucki, W. J., et al. 2010, ApJ, 713, L160

Gough, D. O. 1986, in Hydrodynamic and Magnetodynamic Problems in the Sun and Stars, ed. Y. Osaki, 117

Gough, D. O. 1993, in Astrophysical Fluid Dynamics - Les Houches 1987, eds. J.-P. Zahn, \& J. Zinn-Justin, 399

Jefferies, S. M., Pomerantz, M. A., Duvall, Jr., T. L., Harvey, J. W., \& Jaksha, D. B. 1988, in Seismology of the Sun and Sun-Like Stars, ed. E. J. Rolfe, ESA SP, 286, 279
Jiménez, A. 2006, ApJ, 646, 1398

Jiménez, A., Jiménez-Reyes, S. J., \& García, R. A. 2005, ApJ, 623, 1215

Jiménez, A., García, R. A., \& Pallé, P. L. 2011, ApJ, 743, 99

Kallinger, T., De Ridder, J., Hekker, S., et al. 2014, A\&A, 570, A41

Kumar, P. 1993, in GONG 1992. Seismic Investigation of the Sun and Stars, ed. T. M. Brown, ASP Conf. Ser., 42, 15

Kumar, P., Duvall, Jr., T. L., Harvey, J. W., et al. 1990, in Progress of Seismology of the Sun and Stars, eds. Y. Osaki, \& H. Shibahashi (Berlin: Springer Verlag), Lect. Notes Phys., 367, 87

Libbrecht, K. G. 1988, ApJ, 334, 510

Mathur, S., García, R. A., Régulo, C., et al. 2010, A\&A, 511, A46

Mathur, S., Hekker, S., Trampedach, R., et al. 2011, ApJ, 741, 119

Morel, P., \& Lebreton, Y. 2008, Ap\&SS, 316, 61

Pires, S., Mathur, S., García, R. A., et al. 2015, A\&A, 574, A18

White, T. R., Bedding, T. R., Stello, D., et al. 2011, ApJ, 743, 161 


\section{Appendix A: Figures of the other stars}
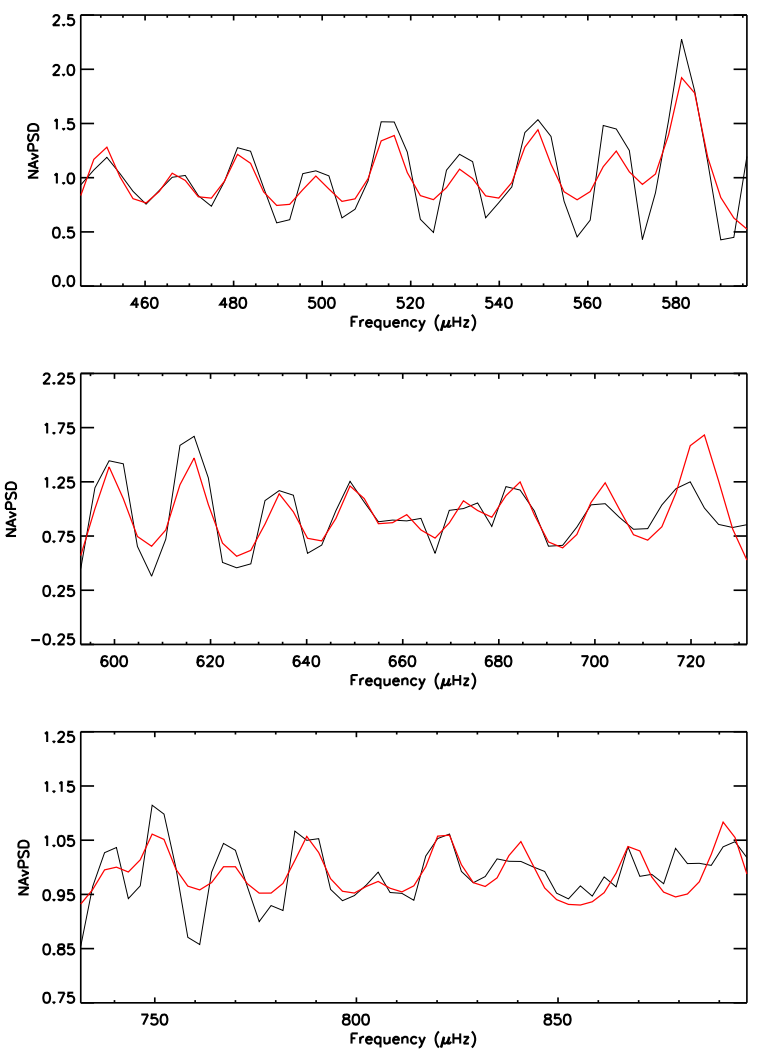

Fig. A.1. Fitted (red line) NAvPSD of KIC 7799349 by groups of nine peaks in the low-frequency range ( $p$-modes).

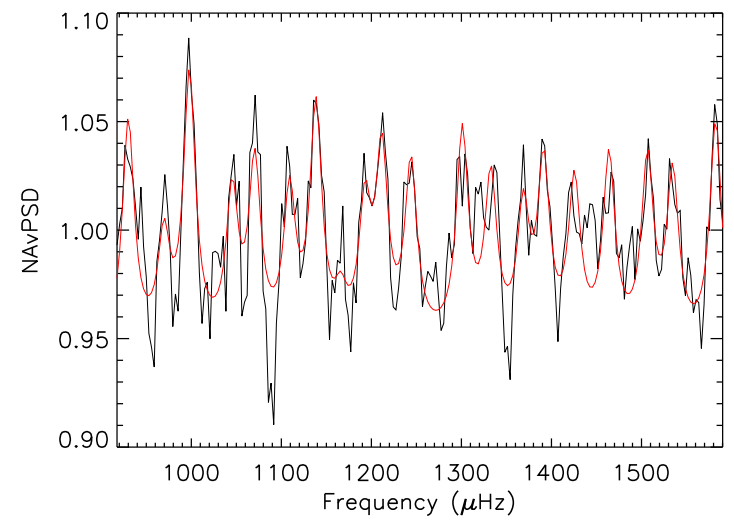

Fig. A.2. Fitted (red line) NAvPSD of KIC 7799349 in the highfrequency range (pseudo-modes).

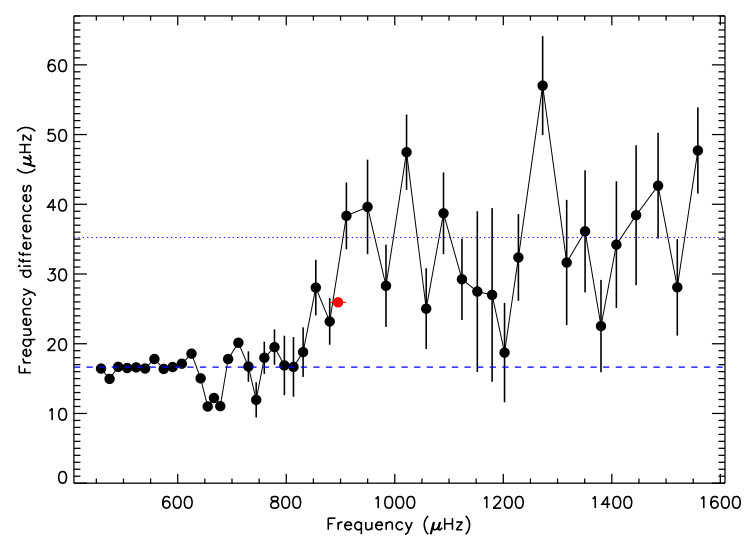

Fig. A.3. Consecutive frequency differences (KIC 7799349); that is, the separations between the fitted peaks for NAvPSD. Two levels are shown: one around $\Delta v / 2$, corresponding to $p$-modes (blue dashed line with a weighted mean of $16.64 \pm 0.09 \mu \mathrm{Hz}$ ), and other around $\Delta v$, corresponding to pseudo-modes (blue dotted line with a weighted mean of $35.23 \pm 1.52 \mu \mathrm{Hz})$. The red symbol is the estimate of the acoustic cut-off frequency $(895.47 \pm 15.38 \mu \mathrm{Hz})$.
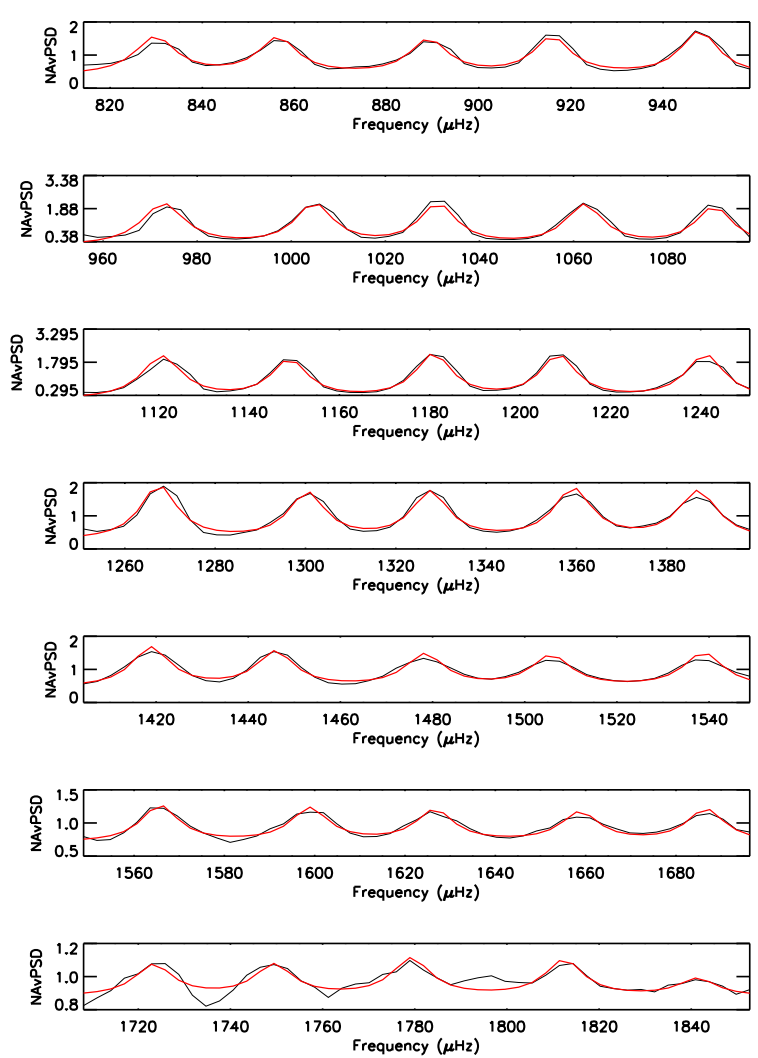

Fig. A.4. Fitted (red line) NavPSD of KIC 7940546 by groups of five peaks in the low-frequency range ( $p$-modes). 
A. Jiménez et al.: Acoustic cut-off frequency and HIPs with Kepler

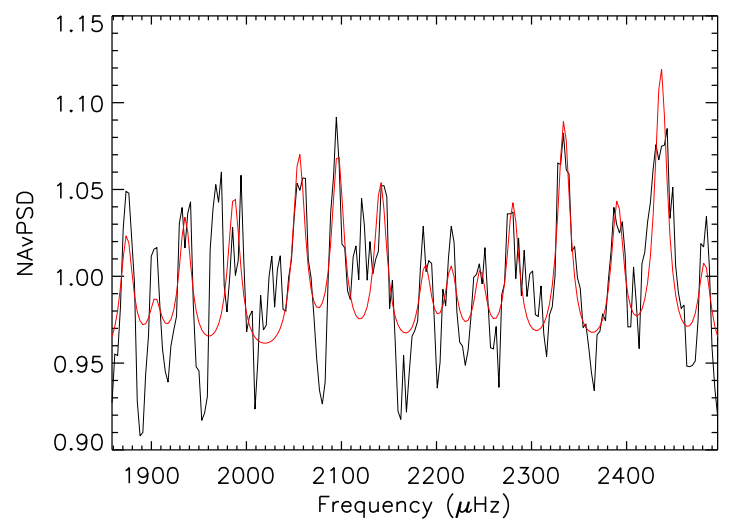

Fig. A.5. Fitted (red line) NAvPSD of KIC 7940546 in the highfrequency range (pseudo-modes).

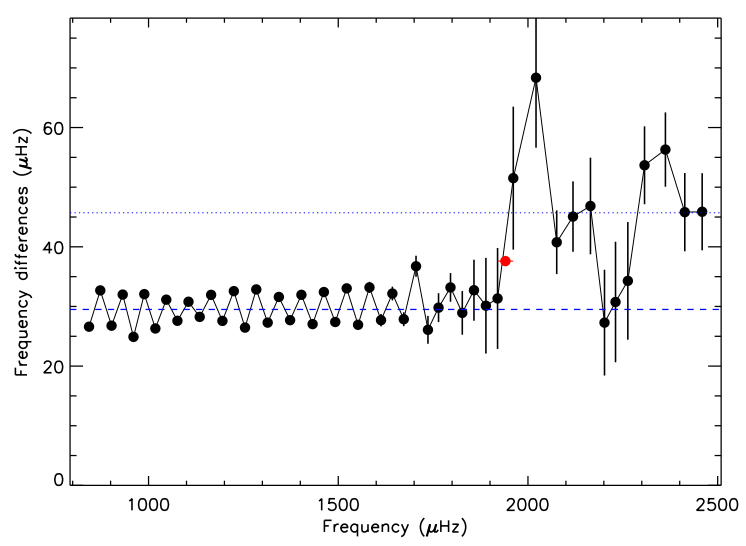

Fig. A.6. Consecutive frequency differences (KIC 7940546), that is, the separations between the fitted peaks for NAvPSD. Two levels are shown: one around $\Delta v / 2$, corresponding to $p$-modes (blue dashed line with a weighted mean of $29.50 \pm 0.07 \mu \mathrm{Hz}$ ), and other around $\Delta v$, corresponding to pseudo-modes (blue dotted line with a weighted mean of $45.71 \pm 2.12 \mu \mathrm{Hz})$. The red symbol is the estimate of the acoustic cut-off frequency $(1940.59 \pm 20.71 \mu \mathrm{Hz})$.
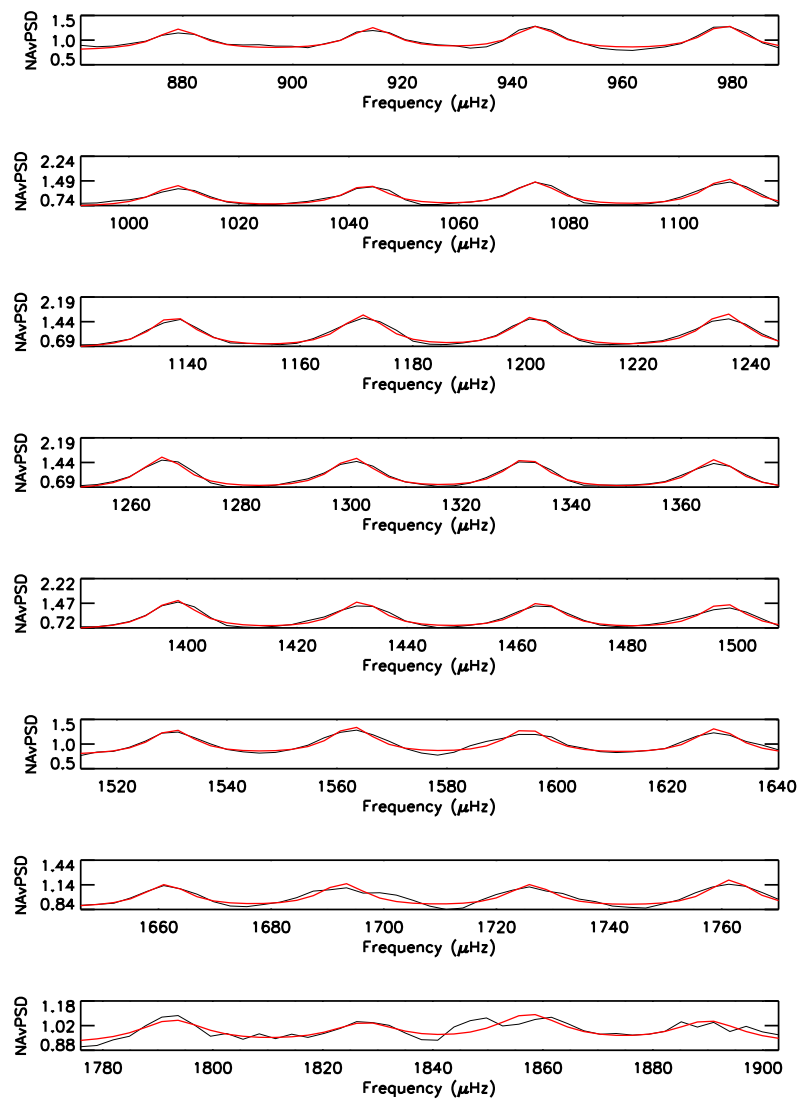

Fig. A.7. Fitted (red line) NAvPSD of KIC 9812850 by groups of four peaks in the low-frequency range ( $p$-modes).

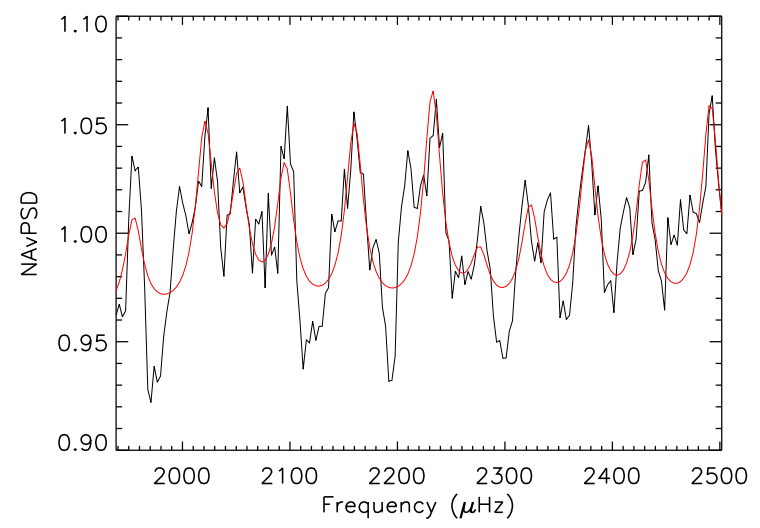

Fig. A.8. Fitted (red line) NAvPSD of KIC 9812850 in the highfrequency range (pseudo-modes). 


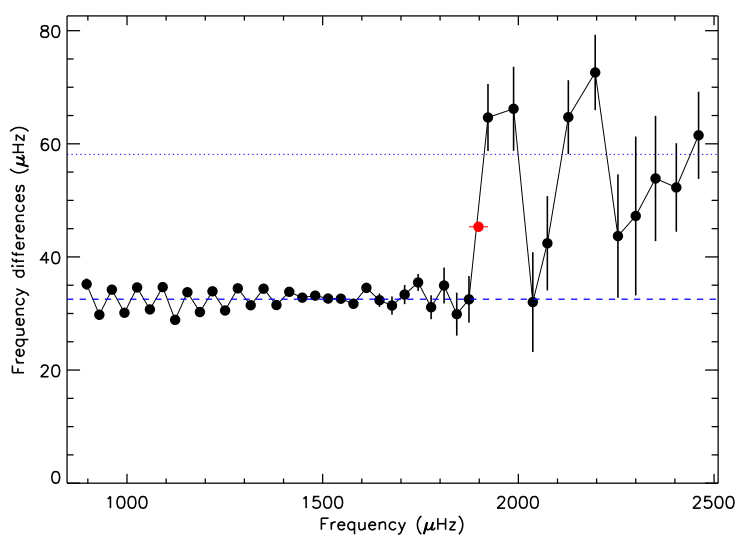

Fig. A.9. Consecutive frequency differences (KIC 9812850); that is, the separations between the fitted peaks for NAvPSD. Two levels are shown: one around $\Delta v / 2$, corresponding to $p$-modes (blue dashed line with a weighted mean of $32.53 \pm 0.12 \mu \mathrm{Hz}$ ), and other around $\Delta v$, corresponding to pseudo-modes (blue dotted line with a weighted mean of $58.13 \pm 2.39 \mu \mathrm{Hz})$. The red symbol is the estimate of the acoustic cut-off frequency $(1898.08 \pm 24.29 \mu \mathrm{Hz})$.
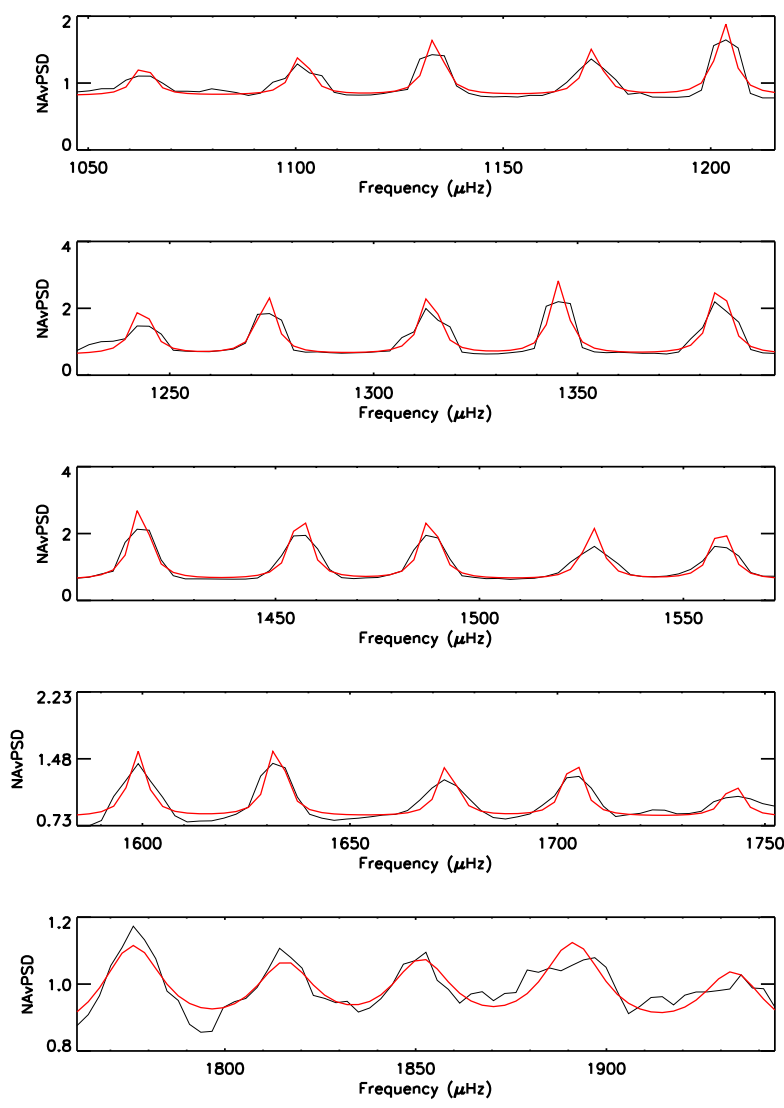

Fig. A.10. Fitted (red line) NAvPSD of KIC 11244118 by groups of five peaks in the low-frequency range ( $p$-modes).

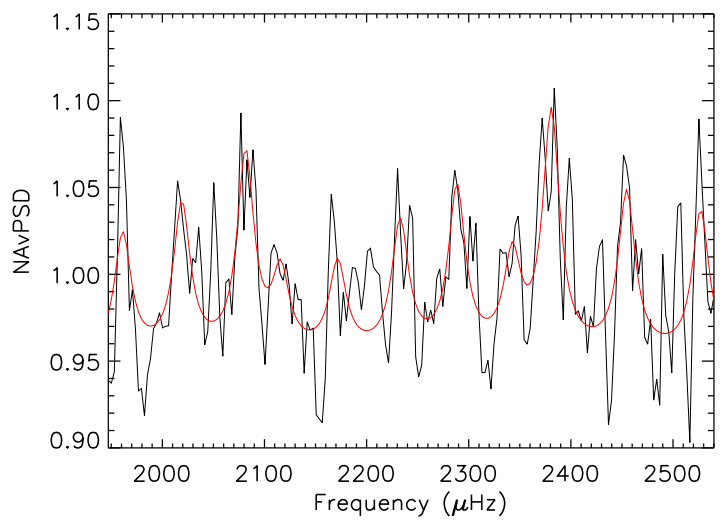

Fig. A.11. Fitted (red line) NAvPSD of KIC 11244118 in the highfrequency range (pseudo-modes).

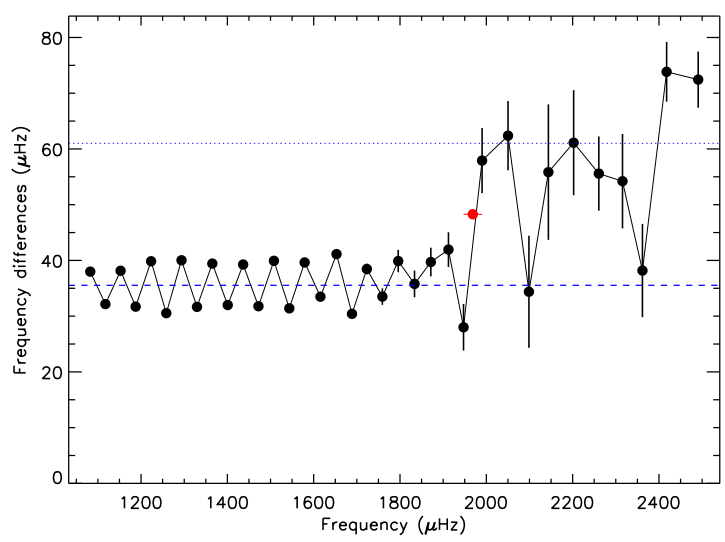

Fig. A.12. Consecutive frequency differences (KIC 11244118); that is, the separations between the fitted peaks for NAvPSD. Two levels are shown: one around $\Delta v / 2$, corresponding to $p$-modes (blue dashed line with a weighted mean of $35.54 \pm 0.08 \mu \mathrm{Hz}$ ), and other around $\Delta v$, corresponding to pseudo-modes (blue dotted line with a weighted mean of $61.02 \pm 2.19 \mu \mathrm{Hz})$. The red symbol is the estimation of the acoustic cut-off frequency $(1968.69 \pm 21.48 \mu \mathrm{Hz})$. 
A. Jiménez et al.: Acoustic cut-off frequency and HIPs with Kepler
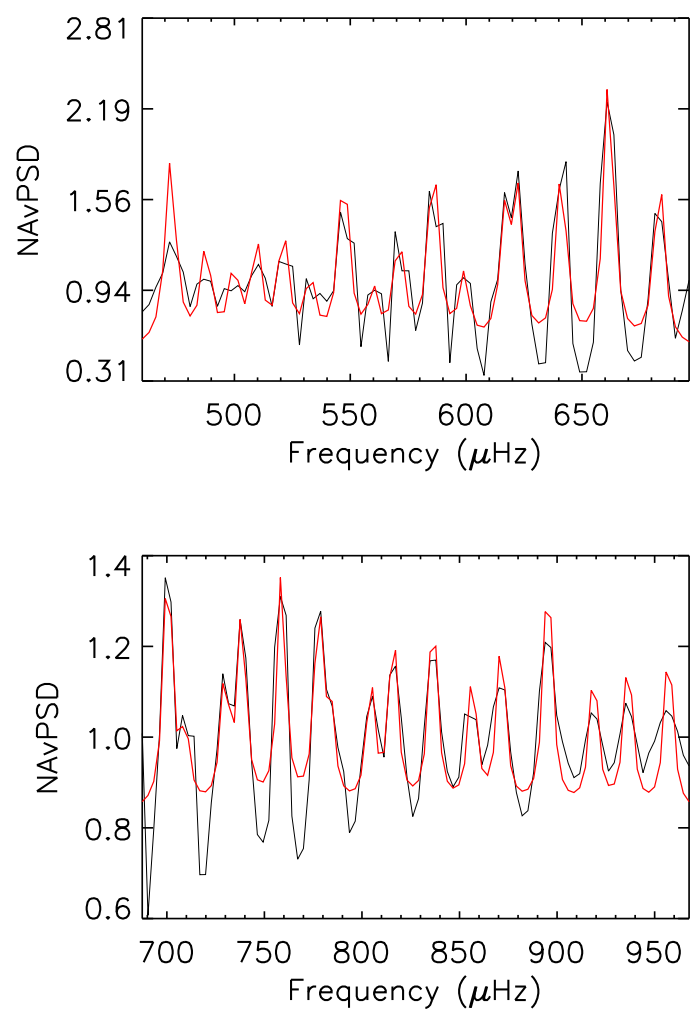

Fig. A.13. Fitted (red line) NAvPSD of KIC 11717120 by groups of sixteen peaks in the low-frequency range ( $p$-modes).

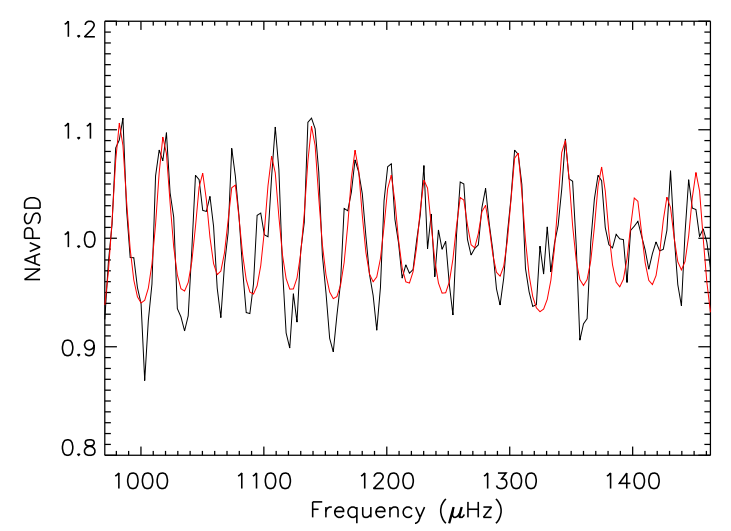

Fig. A.14. Fitted (red line) NAvPSD of KIC 11717120 in the highfrequency range (pseudo-modes).

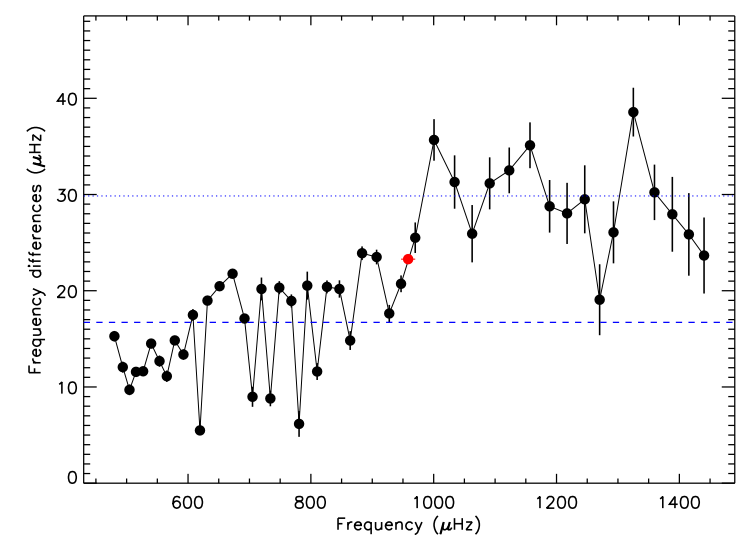

Fig. A.15. Consecutive frequency differences (KIC 11717120); that is, the separations between the fitted peaks for NAvPSD. Two levels are shown: one around $\Delta v / 2$, corresponding to $p$-modes (blue dashed line with a weighted mean of $16.71 \pm 0.09 \mu \mathrm{Hz}$ ), and other around $\Delta v$, corresponding to pseudo-modes (blue dotted line with a weighted mean of $29.85 \pm 0.66 \mu \mathrm{Hz}$ ). The red symbol is the estimation of the acoustic cut-off frequency $(958.38 \pm 11.56 \mu \mathrm{Hz})$ 